\title{
ON SOLUTIONS WITH ALGEBRAIC CHARACTER OF LINEAR PARTIAL DIFFERENTIAL EQUATIONS(1)
}

\author{
BY \\ STEFAN BERGMAN
}

Introduction. One of the methods for the study of harmonic functions $h(x, y)$ of two variables consists in associating with every $h$ a function $f$ of one complex variable by the relation

$$
h(x, y)=2^{-1}[f(x+i y)+f(x-i y)] .
$$

The function $f$ can be obtained by continuing $h(x, y)$ to the complex values of the arguments and then considering it in the characteristic plane $x=-i y$ (or $x=i y$ ): substituting $x=z / 2, y=-i z / 2$ into $2 h(x, y)$ yields $f(z)+$ const.

In the present paper we give a generalization of this method to harmonic functions of three variables. In particular, we study certain classes of harmonic functions $H(\boldsymbol{X}), \boldsymbol{X}=(x, y, z)\left({ }^{2}\right)$. In addition we shall also consider certain classes of functions $\Phi(X)$ which satisfy

$$
T(\Phi) \equiv \Delta \Phi+C\left(x^{2}+y^{2}+z^{2}\right) \Phi=0 .
$$

Here $C\left(x^{2}+y^{2}+z^{2}\right)$ is an entire function of $\left(x^{2}+y^{2}+z^{2}\right)$. In Chapter I we associate with each harmonic function $H(X)$ an analytic function $\chi\left(Z, Z^{*}\right)$ of two variables $Z=(i y+z)^{\prime} / 2$ and $Z^{*}=(i y-z) / 2$ which essentially coincides with $H$ in the characteristic space $\left(^{3}\right) \mathcal{C}_{3}=\mathrm{E}\left[x^{2}+y^{2}+z^{2}=0\right]\left({ }^{4}\right)$.

The transition from the space $\mathfrak{S}$ of functions $\chi\left(Z, Z^{*}\right)$ to the space $\mathfrak{S}$ of harmonic functions $H(X)$ can be realized by certain integral operators, and it is useful for many purposes to insert an intermediary space $\mathfrak{B}$ of functions $f(u, \zeta)$ of two complex variables $u=\left(x+\zeta Z+\zeta^{-1} Z^{*}\right)$ and $\zeta$, and to consider the mappings of $\mathfrak{S}$ onto $\mathfrak{B}$ and $\mathfrak{B}$ onto $\mathfrak{S}$ separately.

In $\$$ I.3 we determine the operator transforming the function $\chi\left(Z, Z^{*}\right)$ into the function $f(u, \zeta)$ and the operator transforming $f(u, \zeta)$ back into $H(\boldsymbol{X})$.

Algebraic harmonic functions are studied in Chapter II, and in particular those which arise from a rational $f$. The latter are single valued on $\mathrm{R}$-manifolds

Presented to the Society, December 29, 1949; received by the editors March 18, 1949.

( $\left.{ }^{1}\right)$ Paper done under contract with the Office of Naval Research NR 043-046.

(2) We shall of ten use the notation $H(X)$ for $H(x, y, z)$.

(3) The solutions $H(x, y, z)$ are in the present paper considered in most cases for real values of the arguments. We sometimes continue them, however, to complex values; in particular, $C_{3}$ is a manifold in the space of three complex variables $x=x_{1}+i x_{2}, y=y_{1}+i y_{2}, z=z_{1}+i z_{2}$, see $\S \mathrm{I} .2$.

$\left.{ }^{4}\right) \mathrm{E}[\cdots]$ denotes the set of points whose coordinates satisfy the relations indicated in the bracket. 
(three-dimensional analogues of Riemann surfaces), and we determine in $\S I I .3$ the maximum number of independent constants possessed by such a function of a given order. This is analogous to one case of the RiemannRoch Theorem.

Chapter III continues these considerations to harmonic vectors $H$, that is, vectors satisfying div $\boldsymbol{H}=0$ and curl $\boldsymbol{H}=0$. Considering the integrals $\int \boldsymbol{H} \cdot d \boldsymbol{X}$, where $\boldsymbol{H}$ is a harmonic vector, we show that certain linear combinations of these integrals equal linear combinations of integrals of algebraic functions of one complex variable; here algebraic relations between the limits of the integrals on both sides exist. (This result represents to a certain extent an analogue of Abel's theorem.)

In Chapter IV we discuss operators transforming harmonic functions into solutions of $\mathbf{T}(\Phi)=0$. By means of these operators we study solutions of $\mathbf{T}(\Phi)=0$ which in the characteristic space $\mathrm{E}\left[x^{2}+y^{2}+z^{2}=0\right]$ are algebraic functions of the arguments $y$ and $z$. We show that these functions are multivalued and are defined on certain $\mathrm{R}$-manifolds $\mathcal{S}$ (closely related with the $\mathrm{R}$-manifolds for harmonic functions considered in II). These functions possess the property that they can be represented in the form

$$
\Phi(\boldsymbol{X})=G(\boldsymbol{X})+\sum_{n=1}^{\infty} B^{(n)}\left(r^{2}\right) G_{n}(\boldsymbol{X}),
$$

where $B^{(n)}\left(r^{2}\right), n=1,2, \cdots$, are certain functions depending only upon the function $C$ in $\mathbf{T}$ and $G(\boldsymbol{X})$ and $G_{n}(\boldsymbol{X}), n=1,2, \cdots$, are functions harmonic and single-valued on $\delta$. We can associate with $\delta$ certain $\tilde{\theta}$ functions, generalization of classical $\theta$-functions; and using these $\theta$-functions, their derivatives, finitely many transcendental functions connected with $\mathcal{S}$, and finitely many algebraic and logarithmic operations, each of the functions $G_{n}(\boldsymbol{X}), n=1$, $2, \cdots$, can be represented in a closed form.

As it has been shown in $[4,7](5)$ the theory of Riemann surfaces of functions satisfying a partial differential equation $L_{2}(\phi) \equiv \Delta \phi+a \phi_{x}+b \phi_{y}+c$ $=0$ is in a very simple manner connected with the theory of Riemann surfaces for harmonic functions. It seems that a similar situation takes place in the three-dimensional case, and that many results obtained for multivalued harmonic functions can be extended to the theory of functions satisfying linear partial differential equations of the form $\Delta \phi+K \phi=0$, where $K$ is an entire function.

The author wishes to thank Mr. Henry Pollak and Mr. Arthur Zeichner for their help in the preparation of the present paper.

\section{INTEGRAL OPERATORS IN THE THEORY OF HARMONIC FUNCTIONS}

1. Preliminaries. The method of integral operators is one of the tools used

${ }^{(5)}$ See also Trans. Amer. Math. Soc. vol. 62 (1947) pp. 452 ff. Numbers in brackets refer to the bibliography. 
to extend procedures of the theory of functions of a complex variable to other fields, in particular to the theory of functions satisfying linear partial differential equations. The totality $\{U\}$ of solutions of a linear partial differential equation represents a linear space. On the other hand, in contrast to functions of a complex variable, the $U$ 's neither form an algebra nor possess group properties. The fact that analytic functions of a complex variable form an algebra gives the basis for the development of the theory of algebraic functions of a complex variable which can be formulated as a theory of algebraic harmonic functions in two variables. One of the aims of the operator theory is to provide some substitutes for operations which permit us to develop the above theory. By associating, in a one-to-one way, a function $f_{k}$ of one or more complex variables with every solution $U_{k}=\mathbf{P}\left(f_{k}\right)$ of a differential equation, we define a certain composition rule " $\otimes$ " for the $U_{k}$ by writing $\left({ }^{6}\right)$

$$
\begin{aligned}
& U_{3}=U_{1} \otimes U_{2}, \\
& U_{3}=\mathbf{P}\left(f_{1} f_{2}\right) .
\end{aligned}
$$

(The function $f_{k}$ corresponding to $U_{k}$ will be denoted as the "P-associate $\left({ }^{7}\right)$ of $U_{k}$. ") On the other hand, the relations like (1.1) are at first purely formal, since the functions $U_{3}$ in (1.1) depend upon the correspondence principle applied, that is, upon the choice of $\mathbf{P}$, and $\mathbf{P}$ can be defined in infinitely many different ways. One of the main problems of the theory of integral operators is to study operators which transform functions $f$ into solutions $U$ and to select among them those which preserve some basic properties of the functions to which the operator is applied. In this way we obtain operators which can be considered as more or less a natural extension of the operation "taking the real part."

Most of the properties of analytic functions can be interpreted as theorems on harmonic functions of two variables. As it has been shown in a number of previous papers, the theory of integral operators (of the first kind) permits us to extend many of these theorems to the case of general linear partial differential equations in two variables of the form $\mathrm{L}_{2}$ (see p. 462).

In attempting to generalize methods of integral operators to differential equations in three variables we find it useful to distinguish between two different problems.

I. Applying operators to analytic functions of one (or more) complex

( $)$ We omit here a more detailed discussion of the opera tions which can be carried out in the theory of functions of one or more complex variables and by employing conveniently chosen operators used in other function-spaces. We restrict ourselves merely to mentioning that $\{f\}$ is an algebra, since this property of $\{f\}$ is used decisively in the study of algebraic harmonic functions in Chapter II. Concerning other types of operators, compare $[6,8,10,17,18]$.

( 7$)$ In order to avoid confusion, it should be stressed that here and in the following operators $\mathbf{P}$ are applied to the associate functions (which in general form an algebra) and transform them into solutions of differential equations in whose properties we are interested. 
variables, we study harmonic functions and harmonic vectors, that is, vectors $H(X), X=(x, y, z)$, for which

$$
\begin{aligned}
& \operatorname{curl} H=0, \\
& \operatorname{div} \boldsymbol{H}=0
\end{aligned}
$$

holds.

II. In a manner analogous to the procedure in two variables, we consider operators transforming harmonic functions in three variables into solutions of more general linear partial differential equations.

In the present paper we study mostly the questions connected with I, and limit ourselves in considering problem II to differential equations of the form

$$
T(\phi) \equiv \frac{\partial^{2} \phi}{\partial x^{2}}+\frac{\partial^{2} \phi}{\partial y^{2}}+\frac{\partial^{2} \phi}{\partial z^{2}}+C\left(r^{2}\right) \phi=0,
$$

where $C$ is an entire function of $r^{2}=x^{2}+y^{2}+z^{2}$.

2. Characteristic space $\mathcal{C}_{3}$. In considering harmonic functions of three variables it is often convenient to continue the arguments $x, y, z$ to complex values and to study their behavior in the characteristic space,

$$
\begin{aligned}
\mathcal{C}_{3} & =\mathrm{E}\left[x^{2}+y^{2}+z^{2}=0\right] \\
& =\mathrm{E}\left[x_{1}^{2}+y_{1}^{2}+z_{1}^{2}=x_{2}^{2}+y_{2}^{2}+z_{2}^{2}, x_{1} x_{2}+y_{1} y_{2}+z_{1} z_{2}=0\right] \\
x & =x_{1}+i x_{2}, \quad y=y_{1}+i y_{2}, \quad z=z_{1}+i z_{2} .
\end{aligned}
$$

Here it is convenient to use coordinates

$$
Z=(i y+z) / 2, \quad Z^{*}=(i y-z) / 2 .
$$

We have then

$$
x=2\left(Z Z^{*}\right)^{1 / 2}, \quad y=-i\left(Z+Z^{*}\right), \quad z=\left(Z-Z^{*}\right) .
$$

LEMMa 1.1. To every harmonic function $\left({ }^{8}\right)$

$$
\begin{aligned}
H=H(X) & =\sum_{n=0}^{\infty} \sum_{k=-n}^{n} 2^{-n}(2 n) ![(n+k) !(n-k) !]^{-1} \alpha_{n k} R^{n} P_{n}^{k}(\cos \theta) e^{i k \phi}, \\
\alpha_{n,-k} & =\bar{\alpha}_{n, k}
\end{aligned}
$$

(which is regular at the origin) there correspond two analytic functions $\chi_{k}\left(Z, Z^{*}\right)$,

${ }^{(8)}$ Here $R, \theta, \phi$ are polar coordinates, and $P_{n}^{k}$ are Legendre functions. We note that in the present paper we use for the Legendre functions $P_{n}^{k}$ the definition adopted in $[11, p .73]$; This definition differs from the definitions used elsewhere by various multiplicative constants. For instance, the $P_{n}^{k}$ used here differ from the $P_{n}^{k}$ used in Madelung, Die mathematischen Hilfsmittel des Physikers by the factor $(-1)^{k}$, see p. 66, and from those in Heine Kugelfunktionen, see p. 211 , by the factor $2^{-n}(2 n) ![n !(n-k) !]^{-1}$. 
$k=1,2$, of two complex variables which are regular at the origin and such that

$$
\begin{aligned}
H\left(2\left(Z Z^{*}\right)^{1 / 2},-i\left(Z+Z^{*}\right),\left(Z-Z^{*}\right)\right) & =\chi\left(Z, Z^{*}\right) \\
& \equiv \chi_{1}\left(Z, Z^{*}\right)+\left(Z Z^{*}\right)^{1 / 2} \chi_{2}\left(Z, Z^{*}\right),
\end{aligned}
$$

where

$$
\chi_{1}=\sum_{n=0}^{\infty} \sum_{k=-n}^{n} \beta_{n k} Z^{(n-k) / 2} Z^{*(n+k) / 2}=\sum \sum \gamma_{\mu \nu} Z^{\mu} Z^{* \nu}, \quad n-k \text { even }
$$

and

$$
\chi_{2}=\sum_{n=0}^{\infty} \sum_{k=-n}^{n} \beta_{n k} Z^{(n-k+1) / 2} Z^{*(n+k-1) / 2}=\sum \sum \gamma_{\mu \nu}^{*} Z^{\mu} Z^{* \nu}, \quad n-k o d d
$$

Here

$$
\beta_{n k}=\alpha_{n k}(2 n) ![(n+k) !(n-k) !]^{-1} ; \gamma_{\mu \nu}=\beta_{\nu+\mu, \nu-\mu}, \gamma_{\mu \nu}^{*}=\beta_{\nu+\mu+1, \nu-\mu} .
$$

Conversely, to every pair of functions $\left(\chi_{1}, \chi_{2}\right)$ there corresponds a harmonic function $H(X)$ related to $\left(\chi_{1}, \chi_{2}\right)$ by the relation (1.10).

We shall denote the harmonic function $H(X)$ corresponding to a given function $\chi\left(Z, Z^{*}\right)$ by $\mathrm{d}_{3}(\chi)$.

Proof. Every real harmonic function $H$ which is regular in the neighborhood $\mathcal{N}(0)$ of the origin can be represented in $\mathcal{N}(0)$ in the form (1.9).

Using the Laplace representation we obtain (see $[11$, p. 211])

$$
H=\frac{1}{2 \pi i} \int_{\mathcal{L}} \sum_{n=0}^{\infty} \sum_{k=-n}^{n} \alpha_{n k} u^{n} \zeta^{k-1} d \zeta
$$

where

$$
u=x+Z \zeta+Z^{*} \xi^{-1}, \quad \mathcal{L}=\mathrm{E}(|\zeta|=1)
$$

Replacing $x$ by $2\left(Z Z^{*}\right)^{1 / 2}$ yields $u=\left((Z \zeta)^{1 / 2}+\left(Z^{*} \zeta^{-1}\right)^{1 / 2}\right)^{2}$, so that the relation (1.10) holds.

The inequalities $\left|\beta_{n k}\right|<c r^{-n}, n=1,2, \cdots$, imply $\left|\gamma_{\mu \nu}^{*}\right|<c r^{-(\nu+\mu)}$, and $\left|\gamma_{\mu \nu}^{*}\right|<c r^{-(\nu+\mu+1)}, \nu+\mu=1,2, \cdots$, and vice versa. Further

$$
1 \leqq(2 n) ![(n+k) !(n-k) !]^{-1} \leqq 4^{n}
$$

and therefore

$$
\left|\alpha_{n k}\right| \leqq\left|\beta_{n k}\right| \leqq 4^{n}\left|\alpha_{n k}\right|
$$

Thus the regularity of $H$ in the neighborhood of the origin implies the regularity of $\chi_{1}$ and $\chi_{2}$ in the neighborhood of the origin and vice versa. This completes the proof of the lemma. 
Definition 1.1. The function $\chi\left(Z, Z^{*}\right)$ will be defined as the $\mathrm{C}_{3}$-associate of $H$.

$$
\mathfrak{\mho}_{3}=\left\{\chi\left(Z, Z^{*}\right)\right\}
$$

forms an algebra. On the other hand, there are certain differences between the situation in the case of $h(x, y)$ and that of $H(X)$ which make it impossible to make an immediate generalization to the three-dimensional case of the procedures used in the theory of harmonic functions $h(x, y)$ of two variables.

1. While the transition from $f(z), z=x+i y$, to $h(x, y)$ is realized by the operator "taking the real part" which will be denoted by $b_{2}$, the operators transforming $\{\chi\}$ into $\{H\}$ known at present are of more complicated nature. The "simplest" operator (which is also considered in the present paper) involves a double integration $\left({ }^{9}\right)$.

2. While in the case of operators $\mathbf{b}_{2}$ and $\mathbf{p}_{2}\left({ }^{10}\right)$, the associates are again functions of two real variables $x$ and $y$, in the three-dimensional case the associate $\chi\left(Z, Z^{*}\right)$ is a function of four real variables, while $H(\boldsymbol{X})$ depends only on three variables.

3. $\chi\left(Z, Z^{*}\right)$ is not analytic at the origin; this deficiency can be easily removed, say by introducing new variables, but then some new complications will arise. Further, $\chi\left(Z, Z^{*}\right)$ are functions of two complex variables whose properties are not so well known as those of functions of one complex variable.

These and other differences between the situation in the two- and in the three-dimensional case suggest that in the latter case we introduce in our investigations an additional step. It consists of the use of an intermediary functions space which is an algebra, namely the space $\mathfrak{B}$ of functions $f(u, \zeta)$ of two complex variables $u=x+\zeta Z+\zeta^{-1} Z^{*}$ and $\zeta\left({ }^{11}\right)$. By an integral operator which involves one integration (see (1.15), (1.16), and (3.2)) one obtains then harmonic functions $H(\boldsymbol{X})$ and the harmonic vector $\boldsymbol{H}(\boldsymbol{X})$.

3. The operator $B_{3}$ and its inverse. The generalized Whittaker formula:

$\left({ }^{9}\right)$ It should be noted that there exist other operators, see e.g. [8], transforming the functions $\chi$ into harmonic functions $H(\mathbf{X})$. Those known at present involve at least a double integration. It is of interest to determine and to study various operators transforming $\{x\}$ (or related linear spaces) into $\{H\}$ since for particular purposes one or another operator may be more appropriate.

(10) To every equation $\mathrm{L}_{2}(\phi) \equiv \Delta \phi+a \phi_{x}+b \phi_{y}+c \phi \equiv 0$ it is possible to associate a set of functions $Q^{(\nu)}=Q^{(\nu)}\left(z, z^{*}\right), z=x+i y, z^{*}=x-i y$ so that the operation $p_{2}(g)=Q^{(0)} g(z)+\sum_{\nu=1}^{\infty} Q^{(\nu)}$ $\cdot \int_{0}^{z}(z-\zeta)^{\nu-1} g(\zeta) d \zeta$ transforms analytic functions of a complex variable into complex solutions of $L_{2}(\psi)=0$. See for details [4].

(11) It should be emphasized that this is only one of the possibilities, and the choice of the most useful intermediary function space depends upon the purposes at hand. This whole topic of properties which can be "translated" from one function's space to another, and the mechanics involved in the mapping of function spaces has not been studied sufficiently until the present. 


$$
\begin{aligned}
H(\boldsymbol{X}) & =\mathbf{B}_{3}\left(f, \mathcal{L}, \boldsymbol{X}_{0}\right), & \mathbf{B}_{3}\left(f, \mathcal{L}, \boldsymbol{X}_{0}\right)=\frac{1}{2 \pi i} \int_{\mathcal{L}} f(u, \zeta) d \zeta / \zeta, \\
u & =x+Z \zeta+Z^{*} \zeta^{-1}, & Z=(i y+z) / 2, \\
Z^{*} & =(i y-z) / 2, &
\end{aligned}
$$

represents an operation transforming analytic functions of $f$ of $u$ and $\zeta$ into harmonic functions of three variables. Here $\mathcal{L}$ is a simple closed oriented curve in the $\zeta$-plane, and $U\left(X_{0}\right)=\mathrm{E}\left[\left(x-x_{0}\right)^{2}+\left(y-y_{0}\right)^{2}+\left(z-z_{0}\right)^{2}<\epsilon\right]^{1} ; \epsilon>0$, sufficiently small. We assume for the time being that $\mathcal{L}$ is the unit circle, $\mathcal{L}=\mathrm{E}\left[\zeta=e^{i_{s}}, 0 \leqq s \leqq 2 \pi\right]$, and $X_{0}$ is the origin. For convenience we shall write in this chapter $\mathbf{B}_{3}(f)$ instead of $\mathbf{B}_{3}(f,|\zeta|=1,0)$.

REMARK 1.1. We should like to stress that the operator $\mathbf{B}_{3}$ defines a mapping "in the small" of $\{f\}$ into $\{H\}:(x, y, z)$ varies in a sufficiently small neighborhood of $U\left(X_{0}\right)$, and the functions $f(u, \zeta)$ which are defined in a sufficiently small neighborhood of $u=0$ and for $|\zeta|=1$ are transformed into functions $H(X)$ defined in $U\left(X_{0}\right)$.

The functions $f$ corresponding to a given $H$ will be defined as the " $\mathrm{B}_{3^{-}}$ associates of $H$."

There is a further basic difference between the operator $\mathbf{B}_{3}$ and the operators $\mathbf{b}_{\mathbf{2}}$ and $\mathbf{p}_{\mathbf{2}}$ considered in the two-dimensional case. While $\mathbf{p}_{\mathbf{2}}$ as well as $\mathbf{b}_{\mathbf{2}}$ defines a one-to-one mapping of functions $f$ into $h$ 's this is not the case for $\mathbf{B}_{3}$. Indeed, one easily sees that

$$
\int \mathcal{L}^{u^{n} \zeta^{k-1} d \zeta=0,} \quad|k|>n,
$$

so that to a given $H(X)$ the associate $f(u, \zeta)$ is determined only within a null-associate

$$
n(u, \zeta)=\sum_{n=0}^{\infty} \sum_{|k|>n} a_{n k} u^{n} \zeta^{k-1},
$$

where $a_{n k}$ are constants.

Thus, using the operator $\mathbf{B}_{3}$ in studying harmonic functions of three variables, one can associate with a given $H(\boldsymbol{X})$ a whole class of functions,

$$
f(u, \zeta)+n(u, \zeta)
$$

where $n$ is an arbitrary null-associate. Evidently, in different investigations, among associates of a given function $H(\boldsymbol{X})$ (which differ from each other by null-associates) some will be more appropriate than others for the purpose under consideration. This suggests that in using the operator method we employ an indirect approach, and at first determine and study those harmonic 
functions which are generated by associates $f(u, \zeta)$ whose properties are well known, for example, by rational, algebraic, and so on, $\mathrm{B}_{3}$-associates.

In characterizing the classes of harmonic functions corresponding to the above categories of associates it will be useful to take into consideration the behavior of their $\mathrm{C}_{3}$-associates.

As we mentioned there exist for given $H$ infinitely many $\mathrm{B}_{3}$-associates, but there exists one and only one $\mathrm{B}_{3}$-associate whose function element has the form

$$
f(u, \zeta)=\sum_{n=0}^{\infty} \sum_{k=-n}^{n} \alpha_{n k} u^{n} \zeta^{k}
$$

which is defined as the $\mathbf{B}_{3}$-associate "normalized with respect to the origin" or shortly, the "normalized $\mathbf{B}_{\mathbf{3}}$-associate."

LEммA 1.2. The normalized $\mathrm{B}_{3}$-associate $f(u, \zeta)$ of a harmonic function which is regular in a sufficiently small neighborhood $\mathrm{N}(0)$ of the origin is given by

$$
f(u, \zeta)=2 \int_{0}^{1} u^{1 / 2} \frac{d\left[u^{1 / 2} \chi\left(u \zeta^{-1} T^{2}, u \zeta(1-T)^{2}\right)\right]}{d u} d T
$$

where $\chi$ is the $\mathrm{C}_{3}$-associate of $H$. (See (1.13).)

Proof. Let the function element of $H$ be given by (1.10). According to (1.12), (1.10), (1.11a), and (1.11b) the function element of $\chi$ is then

$$
\chi=\sum_{n=0}^{\infty} \sum_{k=-n}^{n} \frac{(2 n) !}{(n+k) !(n-k) !} \alpha_{n k} Z^{(n-k) / 2} Z^{*(n+k) / 2} .
$$

Substituting into the right-hand side of (1.22) $Z=u \zeta^{-1} T^{2}, Z^{*}=u \zeta(1-T)^{2}$ yields for the integrand $I$ of (1.21)

$$
I=\sum_{n=0}^{\infty} \sum_{k=-n}^{n}\left(n+\frac{1}{2}\right) \alpha_{n k} C_{2 n, n+k} u^{n} \zeta^{k}[T(1-T)]^{n}\left[T(1-T)^{-1}\right]^{k} .
$$

Here the $C_{n, m}$ are the binomial coefficients. Since

$$
\left[(2 n+1) C_{2 n, n+k}\right]^{-1}=\int_{0}^{1}[T(1-T)]^{n}\left[T(1-T)^{-1}\right]^{k} d T
$$

the relation (1.21) follows.

REMARK 1.2. From the formulas (1.9), (1.12), (1.15), (1.21) we obtain the identity:

$$
H(X)=\frac{1}{\pi i} \int_{|\zeta|=1}\left[\int_{T=0}^{1} \frac{u^{1 / 2} d\left[u^{1 / 2} \chi\left(u \zeta^{-1} T^{2}, u \zeta(1-T)^{2}\right]\right.}{d u} d T\right] \frac{d \zeta}{\zeta}
$$

where $\chi\left(Z, Z^{*}\right)=H\left(2\left(Z Z^{*}\right)^{1 / 2},-i\left(Z+Z^{*}\right),\left(Z-Z^{*}\right)\right)$ and 


$$
u=x+((i y+z) \zeta / 2)+(i y-z) \zeta^{-1} / 2 .
$$

Since in many considerations in applying the operator methods the inverse operator does not appear at all, it seems advantageous to consider the space $\mathfrak{B}$ as originally given. On the other hand in certain questions of basic importance, for example in investigations of the connection between the regularity domains of $H(X)$ and its $B_{3}$-associate $f(u, \zeta)$, the inverse of $\mathbf{B}_{3}$ is needed; in these investigations we have to return again to the function space $\mathfrak{E}_{3}$.

In particular the question arises of determining the domain( $\left.{ }^{12}\right) G$ in which $\mathbf{P}(f)$ is regular if $f$ is regular in a domain $\mathbb{B}$, and, conversely, where $f$ must be regular if $\mathbf{P}(f)$ is regular in a given domain $\left({ }^{13}\right) \mathscr{B}$.

In the case of the integral operator $b_{2}$ ("taking the real part") and $\mathbf{p}_{2}$ (see p. 466) the corresponding domains of regularity are essentially the same, that is, $\mathbf{b}_{2}(f(z))$ and $\mathbf{p}_{2}(f(z))$ are regular in every simply-connected domain of the real $x, y$-plane in which $f(x+i y)$ is regular, and vice versa.

In the three-dimensional case, the analogous problems are much more involved. In order to illustrate a method of handling this problem, we wish to determine the domain in $\mathcal{C}_{3}$ in which the functions $\chi_{1}\left(Z, Z^{*}\right), \chi_{2}\left(Z, Z^{*}\right)$ must be regular if $H(X)$ is regular in a domain say $\mathbb{B}^{3}$, of the real $x, y, z$ space. We shall denote this domain by $\mathcal{F}^{4}\left(\mathcal{B}^{3}\right)$.

The totality of (four-dimensional) manifolds

$$
\begin{aligned}
S^{4}=\mathrm{E}\left[\left(x_{1}-\xi\right)^{2}+\right. & \left(y_{1}-\eta\right)^{2}+\left(z_{1}-\zeta\right)^{2}=x_{2}^{2}+y_{2}^{2}+z_{2}^{2}, \\
& \left.x_{2}\left(x_{1}-\xi\right)+y_{2}\left(y_{1}-\eta\right)+z_{2}\left(z_{1}-\zeta\right)=0\right],
\end{aligned}
$$

where $(\xi, \eta, \zeta)$ ranges over the boundary $\mathcal{B}^{\prime 2}$ of $\mathcal{B}^{3}$, divides the six-dimensional space of three complex variables, $x=x_{1}+i x_{2}, y=y_{1}+i y_{2}, z=z_{1}+i z_{2}$, into parts. That connected part which includes $\mathcal{B}^{3}$ will form a domain which will be denoted by $\mathcal{F C}^{6}\left(\mathbb{B}^{3}\right)\left({ }^{14}\right)$. The intersections of $S^{4}$ and $\mathcal{F}^{6}\left(\mathcal{B}^{3}\right)$ with the characteristic space $C^{4}=\mathrm{E}\left[x^{2}+y^{2}+z^{2}=0\right]$ will be the manifold

$$
\begin{aligned}
\mathcal{X}^{2}(\Xi)= & \mathrm{E}\left[16 \xi^{2} Z Z^{*}=\rho^{4}-4 \eta^{2}\left(Z+Z^{*}\right)^{2}+4 \zeta^{2}\left(Z-Z^{*}\right)^{2}\right. \\
& +4 i \eta \rho^{2}\left(Z+Z^{*}\right)-4 \zeta \rho^{2}\left(Z-Z^{*}\right)-8 i \eta \zeta\left(Z^{2}-Z^{* 2}\right), \\
\rho^{2}= & \xi^{2}+\eta^{2}+\zeta^{2}
\end{aligned}
$$

and a domain $\mathcal{F}^{4}\left(\mathscr{B}^{3}\right)$, respectively.

(12) It should be noted that if the regularity of $f$ in $\mathbb{B}$ implies the regularity of $\mathbf{P}(f)$ in $G$, the regularity of $g$ in $G$ does not necessarily imply the regularity of $\mathrm{P}^{-1}(g)$ in $B$.

(13) In the remaining part of the present section the superscript over a manifold will indicate its dimension.

${ }^{(14)}$ We wish to mention that N. Aronszajn (Acta Math. vol. 65 (1935)) has already implicitly considered such extensions for harmonic functions of several real variables to the complex domain. 
LEMMA 1.3. Let a harmonic function $H(X)$ be regular in the domain $\mathbb{B}^{3}$ of the three-dimensional $x, y, z$-space, and let

$$
\chi\left(Z, Z^{*}\right)=\chi_{1}\left(Z, Z^{*}\right)+i\left(Z Z^{*}\right)^{1 / 2} \chi_{2}\left(Z, Z^{*}\right)
$$

be the $\mathrm{C}_{3}$-associate of $H$. Then $\chi_{k}\left(Z, Z^{*}\right), k=1,2$, are regular in the domain $\mathfrak{F}^{4}\left(\mathrm{~B}^{3}\right)$ described above.

Proof. The representation

$$
\begin{gathered}
H(X)=-\frac{1}{4 \pi} \iint_{\mathcal{B}^{\prime 2}}\left[H(\Xi) \frac{\partial r^{-1}}{\partial n}-\frac{1}{r} \frac{\partial H(\Xi)}{\partial n}\right] d \omega, \\
r^{2}=\left[(x-\xi)^{2}+(y-\eta)^{2}+(z-\zeta)^{2}\right],
\end{gathered}
$$

of a function $H(\boldsymbol{X})$ which is regular in the domain $\mathcal{B}^{3}+\mathcal{B}^{\prime 2}$, implies that $H(X)$ will be regular when continued to the complex values of the arguments in the domain $\mathcal{F C}^{6}$ of the six-dimensional space. $H\left(x_{1}+i x_{2}, y_{1}+i y_{2}, z_{1}+i z_{2}\right)$ is regular in $\mathbb{B}^{6}$ and therefore $\chi\left(Z, Z^{*}\right)$ considered as a function of $Z^{1 / 2}$ and $Z^{* 1 / 2}$ is also regular in $\mathcal{F}^{4}\left(\mathcal{B}^{3}\right)$, which implies that $\chi_{1}$ and $\chi_{2}$ are regular functions of $Z$ and $Z^{*}$ in $\mathcal{F}^{4}\left(\mathcal{B}^{3}\right)$.

\section{HARMONIC FUnCtIONS With ALGEBRAIC B $_{3}$-ASSOCIATES}

1. General remarks. As we indicated in Chapter I, the method of integral operators enables us to generalize various procedures in the theory of functions of a complex variable to the theory of harmonic functions $H(X)$.

One of the problems of this type is that of developing an analogue of the theory of algebraic functions and their integrals.

Using the theory of analytic functions, we can easily show that algebraic harmonic functions of two variables defined on the Riemann surface

$$
A(\zeta) \equiv \zeta^{N}+\sum_{\nu=1}^{N} a_{\nu}(z) \zeta^{N-\nu} \equiv \zeta^{N}+\sum_{\nu=1}^{N} A_{\nu}(x, y) \zeta^{N-\nu}=0
$$

can be represented in the form

$$
\sum_{\mu=0}^{N-1} \frac{L^{(\mu)}(x, y)}{P^{(\mu)}(x, y)}\left[\zeta^{(\nu)}(x, y)\right]^{\mu}
$$

$\zeta^{(\nu)}(x, y), \nu=1,2, \cdots, N$, being solutions of (2.1). Here $A_{\nu}, L^{(\mu)}, P(\mu)$ are certain polynomials in $x$ and $y$, which can be easily described. For a given $A(\zeta)$ the number of arbitrary constants which appear in $L^{(\mu)}$ and $P^{(\mu)}$ (if their degrees are prescribed) can be determined.

In order to generate algebraic harmonic functions we use in the threedimensional case the operator $B_{3}$.

In $\$ I .2$ we introduced this operator in a general form. We assumed that the integration curve is an arbitrary sufficiently smooth closed curve $\mathcal{L}$ and the integration is carried out for $X \in \mathcal{U}\left(X_{0}\right)=\mathrm{E}\left[\left(x-x_{0}\right)^{2}+\left(y-y_{0}\right)^{2}+\left(z-z_{0}\right)^{2}\right.$ 
$<\epsilon], X_{0}$ being not necessarily the origin, and $\epsilon>0$ being sufficiently small.

In the subsequent considerations of I we specialized $X_{0}$ to be the origin and $\mathcal{L}$ to be a circle with center at $\zeta=0$.

In the development of the approach given in the present paper, it is of importance that we are allowed freedom in the matter of varying the integration curve $\mathcal{L}$. If for the fixed value of $\boldsymbol{X}$ for which the integration is carried out, varying the curve $\mathcal{L}$, we do not intersect any singularities of its $B_{3}$ associate, then the value of the integral remains unchanged. If, in varying $\mathcal{L}$, we meet a pole, the value of the integral increases by its residue.

In the following we shall apply the operator $\mathbf{B}_{3}\left(f, \mathcal{L}, \boldsymbol{X}_{\mathbf{0}}\right)$ in the general form. Varying $\mathcal{L}$ or $X_{0}$ one obtains different harmonic functions, but it is of importance that there exist comparatively simple relations between the harmonic functions obtained from the same associate $f$. In particular, as it has been indicated in [2] in the case of a rational associate $f$ the following results hold.

Let $f=[m(u, \zeta) / q(u, \zeta)]=[M(\zeta ; \boldsymbol{X}) / Q(\zeta ; \boldsymbol{X})]$ where $Q$ is a polynomial in $\zeta$ and $M(\zeta ; X)$ is regular in a sufficiently small domain of the $\zeta, x, y, z$ space. We denote by $\zeta^{(\nu)}(X), \nu=1,2, \cdots, n$, the zeros of $Q\left(\zeta ; X_{0}\right)=0$. Let us assume further that the $\zeta^{(\nu)}\left(X_{0}\right), \nu=1,2, \cdots, n$, differ from each other. If we choose for $\mathcal{L}$ a sufficiently small circle $\mathcal{L}_{\nu}$ with the center at $\zeta^{(\nu)}\left(\boldsymbol{X}_{0}\right)$, then $\left({ }^{15}\right)$

$$
\mathbf{B}_{3}\left(f, \mathcal{L}_{\nu}, \boldsymbol{X}_{0}\right)=\left[\frac{M(\zeta, \boldsymbol{X})}{\partial Q(\zeta, \boldsymbol{X}) / \partial \zeta}\right]_{\zeta \zeta^{(\nu)}(\boldsymbol{X}) .}
$$

$X \in \mathcal{U}\left(X_{0}\right)$

If $\mathcal{L}$ is a closed curve which does not include either the origin $\zeta=0$, nor any of the $\zeta^{(\nu)}\left(X_{0}\right)$, then $\mathbf{B}_{3}\left(f, \mathcal{L}, \boldsymbol{X}_{0}\right)=0$. If finally $\mathcal{L}$ is a sufficiently small circle with center at the origin, and if all $\zeta^{(\nu)}\left(X_{0}\right) \neq 0, \nu=1,2, \cdots, n$, then we obtain a function which is regular in $x, y, z$ in a sufficiently small neighborhood of $X=0$, see $[2$, p. $646 \mathrm{ff}$.].

In the next section we shall indicate the $\mathrm{R}$-manifolds $\left({ }^{16}\right)$ (three-dimensional analogues of Riemann's surfaces) on which these functions are defined. (See (2.14).) Further, in (2.13a), (2.13b) (see also (2.9), (2.5), and (2.25)), we give a representation for them which is analogous to (2.2).

Our procedure can be almost immediately generalized to the case of an algebraic $\mathrm{B}_{3}$-associate. In this case, in addition to algebraic harmonic functions we obtain certain transcendental functions. These functions can be characterized by the property that considered as functions of $x, y$, and $z$,

(15) The idea of generating algebraic harmonic functions by the use of the residue formula from functions $f(u, \zeta)$ was indicated in [1] and [2]. The connection with the space $\bigodot_{3}$ and the extension to more general differential equations were not discussed there. The extension of the Riemann-Roch and the Abel theorems, the problems discussed in $\S \S I I .3$ and II.4, were also not considered there.

${ }^{(16)}$ In order to avoid confusion with Riemann's manifold we use the term R-manifold. The other term has already been used in differential geometry. 
respectively, they satisfy ordinary differential equations with algebraic coefficients. (See [2 pp. $51 \mathrm{ff}$.$] ) Although the formulas arising in the case of$ algebraic associates are more involved, in principle no additional difficulties are encountered in this generalization.

The next class of associates which is of interest in connection with our approach is that of harmonic functions possessing associates which are integrals of the form

$$
f(u, \zeta)=\int_{0}^{1} R(u, \zeta, T, \eta) d T, \quad a(u, \zeta, T, \eta)=0
$$

where $R$ and $a$ are a rational function and a polynomial, respectively $\left({ }^{17}\right)$, in $u, \zeta, T, \eta$. Since $u$ and $\zeta$ can be considered as parameters, the integrals can be expressed in terms of functions connected with functions mapping certain Riemann surfaces on the unit circle or the punctured plane.

The associate $f\left(\left(x+2^{-1} i y\left(\zeta+\zeta^{-1}\right)+2^{-1} z\left(\zeta-\zeta^{-1}\right)\right), \zeta\right)$ considered as a function of $\zeta$ will then again be determined on a Riemann surface (which depends upon the parameters $x, y, z)$ and the study of the corresponding harmonic functions $\int \mathcal{L} f(u, \zeta) d \zeta / \zeta$ can be reduced to certain problems in the theory of functions of a complex variable defined on Riemann surfaces of the above type. The functions obtained will be, in general, transcendental functions which in some special cases become algebraic. It should be stressed that, according to Lemma 1.2, this class of harmonic functions includes all possible algebraic harmonic functions, for if $H(\boldsymbol{X})$ is an algebraic function, then according to (1.27) its associate can be represented in the form (2.3). On the other hand, it has not been clarified as to whether all algebraic harmonic functions can be obtained by merely considering algebraic $\mathrm{B}_{3}$-associates. If this were possible it would permit us to dispense with further study of associate functions of type (2.3) whose theory is comparatively much more involved.

As we indicated in $\S I .3$, in contrast to the two-dimensional case where the $\mathrm{p}_{2}$-associate is uniquely determined and is essentially equal to the value of the function $h(z, \bar{z})$ in the characteristic plane, the $\mathrm{B}_{3}$-associate of $H(X)$ is determined only within a null $\mathrm{B}_{3}$-associate. Therefore, if we make a convenient choice of the null associate we can then possibly obtain a $\mathrm{B}_{3}$-associate

(17) Indeed suppose that $H(\boldsymbol{X})$ is an algebraic harmonic function which is regular at the origin. Then in a sufficiently small neighborhood of the origin it can be developed into a power series (1.9). By (1.23) it can be written in the form $H(X)=(1 / 2 \pi i) \int_{|\zeta|=-f}(u, \zeta) d \zeta / \zeta$ where $f(u, \zeta)$ is given by (1.21). We assume that $H(X)$ is algebraic, that is, we have $H(x, y, z)$ $=R(\eta, x, y, z)$ where $a(\eta, x, y, z)=0 . R$ denotes a rational function and $a$ a polynomial in $\eta$, $x, y, z$. If now we make the substitution (1.8) to obtain the corresponding $\chi\left(Z, Z^{*}\right)$ we see that $\chi\left(Z, Z^{*}\right)=R\left(\eta, 2\left(Z Z^{*}\right)^{1 / 2},-i\left(Z+Z^{*}\right),\left(Z-Z^{*}\right)\right)$ where $\eta$ satisfies the equation: $a\left(\eta, 2\left(Z Z^{*}\right)^{1 / 2}\right.$, $\left.-i\left(Z+Z^{*}\right),\left(Z-Z^{*}\right)\right)=0$, that is, $\chi\left(Z, Z^{*}\right)$ is again an algebraic function. Hence $f(u, \zeta)$ can be represented in the form (2.3), and we could restrict ourselves to the choice of $|\zeta| \doteq 1$ for $\mathcal{L}$. It is, however, in the following, advantageous to use more general curves for $\mathcal{L}$. 
which is of a particularly simple structure. These facts suggest that in the development of this theory an indirect approach be used, consisting of the study of harmonic functions generated by rational, algebraic, $\cdots, \mathrm{B}_{3}$-associates. The characterization of the harmonic functions (by properties of their $\mathrm{C}_{3}$-associates) presents certain difficulties which are caused on the one hand by the existence of null associates and on the other by the fact that in the case of harmonic functions in three dimensions we have to make an integration passing from the $\mathrm{C}_{3}$-associate to the corresponding $\mathrm{B}_{3}$-associate of a given harmonic function $H$ (while the $\mathrm{p}_{2}$-associate is essentially equal in value to the function in the characteristic plane).

In the considerations of $\$ 2-\$ 4$, we shall assume that $\mathcal{L}$ is the circle $\mathcal{L}$, and that the pole under consideration is of the first order, so that the $v$ alue of the residue of $f=P(\zeta ; X) / Q(\zeta ; X)$ has the simple form $2 \pi i P(\zeta ; X)$ $\cdot[\partial Q(\zeta ; X) / \partial \zeta]^{-1}$.

2. An explicit representation of algebraic harmonic functions with a rational associate. In this section, we shall describe harmonic functions whose associates, $f$, are rational functions, that is, for whose associates we have

$$
f(u, \zeta)=\frac{p(u, \zeta)}{q(u, \zeta)}
$$

where $p$ and $q$ are polynomials in $u$ and $\zeta$ and $\zeta^{-1}$. As is shown in Theorem 2.1 , we obtain, in general, algebraic harmonic functions, $H(X)$, which in some special instances reduce to rational functions.

The $H(X)$ 's are rational functions $R\left(\zeta^{(\nu)}, X\right)$ of four variables $\zeta^{(v)}, x, y, z$ where $\zeta^{(\nu)}$ and $x, y, z$ are connected by the equation (2.14). This latter equation (like (2.1) in the two-dimensional case) defines the R-manifold $R$, on which the $R\left(\zeta^{(v)}, X\right)$ are defined and single-valued $\left({ }^{18}\right)$. Since the right-hand side of (2.14) is $q(u, \zeta)$ written in somewhat different form, $\mathbb{R}$ depends only on $\zeta^{(v)}$.

As we shall show, the $H(X)=R\left(\zeta^{(v)}, X\right)$ obtained by integration from (2.4) can be represented (in analogy to (2.2)) in the form (2.13a) or (2.13b) where $\zeta^{(v)}(X)$ is a root of $(2.14) . A_{0}, A_{2 N}$, and $Q$ are polynomials which depend only upon the R-manifold $R$, while the polynomials $G_{\alpha}$ and $L^{(\mu)}$ as well as the integers $M$ and $m$ can vary if $p(u, \zeta)$ varies, that is, if $R\left(\zeta^{(\nu)}, X\right)$ ranges over

(18) It should be noted that the $H(X)=R\left(\zeta^{(\nu)}, X\right)$ can be considered either for complex, or for real values only, of the arguments $x, y, z$. In the first case, the R-manifolds are defined in six-dimensional space, and the considerations lead to a simpler and more unified theory. In the second case we consider the intersection of the above mentioned (six-dimensional) R-manifolds with the real space. This leads to the fact that certain degenerate cases appear, for example, $\left(x^{2}+y^{2}+z^{2}\right)^{-1 / 2}$, which is a two-valued function in the complex space, becomes single-valued in the real space.

Since in the applications we are primarily interested in the behavior of functions for real values of the arguments, we consider $H(\boldsymbol{X})$ in the present paper in the space of three real variables. 
the totality of functions defined on $R$ which are generated by our procedure.

In order to obtain the desired representations $(2.13 \mathrm{a}),(2.13 \mathrm{~b})$ we have to replace $u$ in $p$ and $q$ by $x+(i y / 2)\left(\zeta+\zeta^{-1}\right)+z\left(\zeta-\zeta^{-1}\right) / 2$ and write $p$ and $q$ as polynomials in $\zeta$ with coefficients $G_{\nu} \equiv G_{\nu}(X)$ and $A_{\nu} \equiv A_{\nu}(X)$, respectively. These coefficients are certain combinations of the polynomials $\Gamma_{\nu, \lambda}$ introduced in (2.6).

The connection between analytic functions of a complex variable and harmonic functions of two variables provides in many instances a very elegant representation for the latter functions belonging to some particular class.

There does not exist in the theory of harmonic functions of three variables a direct substitute for the above representations, and we must introduce symbols and develop a certain apparatus to represent conveniently algebraic functions $R\left(\zeta^{(v)}, X\right)$.

Notation. 2.1. A number in brackets, say $[s]$, means the smallest integer which is larger than or equal to $s$.

2.2. $\Gamma_{\nu, \lambda}, \lambda \leqq 2 \nu$, denotes a homogeneous polynomial

$$
\Gamma_{\nu, \lambda}=\Gamma_{\nu, \lambda}(X)=\sum_{j=[\lambda / 2]}^{\min (\lambda, \nu)} 2^{-\nu} C_{\nu, j} C_{j, \lambda-j}(i y-z)^{\lambda-i}(2 x)^{2 j-\lambda}(i y+z)^{\lambda-i}
$$

$\lambda \leqq 2 \nu$

Here $C_{m, n}$ denotes the binomial coefficient.

EXAMPLES. $\Gamma_{00}=1, \quad \Gamma_{\nu, 0}=2^{-\nu}(i y-z)^{\nu}, \quad \Gamma_{\nu, 1}=2^{-\nu+1}(i y-z)^{\nu-1} x, \quad \Gamma_{\nu, 2}=\nu 2^{-\nu}$ $\cdot(i y-z)^{\nu-2}\left(2(\nu-1) x^{2}-y^{2}-z^{2}\right), \cdots, \Gamma_{\nu, 2 \nu}=2^{-\nu}(i y+z)^{\nu}$.

REMARK 2.1. $\Gamma_{\nu, \lambda}$ is a homogeneous polynomial of degree $\nu$ in $x, y, z$.

REMARK 2.2. We note that in the characteristic subspace $\mathcal{C}_{3}$ (see p. 464), the $\Gamma_{r, \lambda}$ becomes

$$
\Gamma_{\nu, \lambda}\left\{i\left(y^{2}+z^{2}\right)^{1 / 2}, y, z\right\}=C_{2 v, \lambda} Z^{\lambda / 2} Z^{* \nu-\lambda / 2} .
$$

Lemma 2.1. A polynomial $\left({ }^{19}\right)$ in $u, \zeta, \zeta^{-1}$

$$
p(u, \zeta)=\sum_{\nu=0}^{m} u^{\nu}\left(\sum_{s=-(M-\nu)}^{M-\nu} b_{\nu s} \zeta^{s}\right)
$$

can be written in the form

$$
p(u, \zeta)=\zeta^{-M} P(\zeta ; X), \quad P(\zeta ; X) \equiv \sum_{s=0}^{2 M} G_{s} \zeta^{s}
$$

where $\left({ }^{20}\right)$

(19) We note that we have to consider only the case $m \leqq M$, since otherwise the summation $\sum_{\varepsilon-(M-\nu)}^{\alpha-\nu}$, for $\nu=M+1, M+2, \cdots, m$, becomes meaningless, and we replace these terms by 0 .

$\left.{ }^{20}\right)$ If $s=0$, we use only the first term in the right-hand side expressions of (2.9). 


$$
\begin{aligned}
& G_{s}=\sum_{\lambda=0}^{\min ([(\sigma-1) / 2], m)} \sum_{j=\lambda}^{\min (m, M+\lambda-[\theta / 2])} b_{j,-M+j+\varepsilon-2 \lambda} \Gamma_{j, 2 \lambda} \\
& +\sum_{\lambda=1}^{\min ([\delta / 2], m)} \sum_{j=\lambda}^{\min (m, M+\lambda-[(8+1) / 2])} b_{j,-M+j+8-2 \lambda+1} \Gamma_{j, 2 \lambda-1} .
\end{aligned}
$$

Proof. A formal computation yields $u^{\nu}=\zeta^{-\nu} \sum_{\lambda=0}^{2 \nu} \Gamma_{\nu, \lambda} \zeta^{\lambda}$, so that the righthand side of $(2.7)$ can be written as

$$
\left.\sum_{\nu=0}^{m} \sum_{\lambda=0}^{2 \nu} \sum_{s=-(M-\nu)}^{N-\nu} \Gamma_{\nu, \lambda} b_{\nu s}\right\}^{s+\lambda-\nu} .
$$

Collecting coefficients of various powers of $\zeta$, say of $\zeta^{-M+2 \sigma}, 0 \leqq \sigma \leqq M$, and of $\zeta^{-M+2 \sigma+1}, 0 \leqq \sigma \leqq M-1$, we consider separately coefficients involving $\Gamma_{\nu, \lambda}$, with odd and those with even $\lambda$, and then we obtain the two terms in the right-hand side of (2.9).

CoROLlary 2.1. Analogously to the representation (2.9) of $p$ we can represent the polynomial in $u, \zeta, \zeta^{-1}$

$$
q(u, \zeta)=\sum_{\nu=0}^{n} u^{\nu}\left(\sum_{s=-(N-\nu)}^{N-\nu} a_{\nu 8} \zeta^{s}\right)
$$

in the form

$$
q(u, \zeta)=\zeta^{N} Q(\zeta ; X), \quad Q(\zeta ; X)=\sum_{\nu=0}^{2 N} A_{\nu} \zeta^{\nu}
$$

where the $A_{\nu}$ are built from the $\Gamma_{\nu, \lambda}$ in exactly the same manner as the $G_{\nu}, m, M$, and $b_{k, 8}$ being replaced by $n, N$, and $a_{k, s}$, respectively.

LEMMA 2.2. A rational function in $u$ and $\zeta,(2.4)$, where $p$ and $q$ are given by (2.7) and (2.10) respectively, can be written in the case where ${ }^{(21)} m<n$, in the form

$$
f=\frac{\zeta^{N-M} \sum_{\nu=0}^{2 M} G_{\nu} \zeta^{\nu}}{A_{2 N}\left(\zeta^{2 N}+\sum_{\nu=1}^{2 N} \frac{A_{\nu}}{A_{2 N}} \zeta^{\nu}\right)}=\frac{\zeta^{N-M} P(\zeta, X)}{Q(\zeta, X)}
$$

where $A$, and $G$, are polynomials, described in Lemma 2.1 and Corollary 2.1.

In analogy to the representation (2.2), we obtain

TheOREM 2.1. The harmonic functions $H=\mathrm{B}_{3}\left(f, \mathcal{L}, X_{0}\right)$ (see p. 467) with

(21) If $m \geqq n$, then we can reduce the degree (in $u$ ) of the numerator, by subtracting a polynomial in $u$. 
associates $f$ of the form (2.4) are rational functions $R\left(\zeta^{(v)}, X\right)$ of four variables $\zeta^{(v)}, x, y, z$,

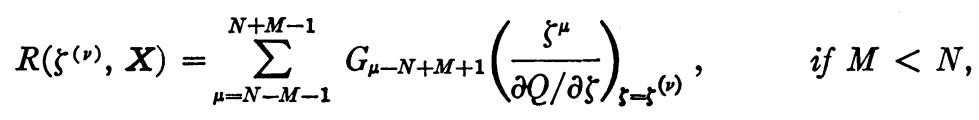

and

$$
R\left(\zeta^{(\nu)}, X\right)=\sum_{\mu=0}^{2 N-1} \frac{L^{(\mu)}}{A_{0}\left(A_{0} A_{2 N}\right)^{M-N}}\left(\frac{\zeta^{\mu}}{\partial Q / \partial \zeta}\right)_{\zeta=\zeta^{(\nu)}}, \quad \text { if } M \geqq N,
$$

where $\zeta^{(v)}$ denotes a root of the equation $\left({ }^{22}\right)$

$$
A(\zeta) \equiv \frac{Q(\zeta, X)}{A_{2 N}} \equiv \zeta^{2 N}+\sum_{\mu=0}^{2 N-1}\left(A_{\mu} / A_{2 N}\right) \zeta^{\mu}=0 .
$$

The $L^{(\mu)}$ are given by (2.25).

REMARK 2.3. The dependence of the $L^{(\mu)}$ upon $b_{v s}$ will be described in Theorem 2.2.

REMARK 2.4. $\mathcal{L}$ does not include the origin, or a double point, as has been discussed previously.

Proof. We introduce at first some new symbols.

Notation. 2.3. Let $S_{\mu}^{(v)}, \mu=1, \cdots, p$, denote a set of positive integers whose sum equals $\nu$. Let $m_{1}^{(\mu)}, \cdots, m_{j}^{(\mu)}, \cdots, m_{n}^{(\mu)}$ be the distinct integers appearing in $S_{\mu}^{(\nu)}, m_{j}^{(\mu)}$ appearing $\sigma_{j}^{(\mu)}$ times. (For instance, a possible $S_{\mu}^{(10)}$ is $(1,1,1,2,2,3)$; in this case $m_{1}^{(\mu)}=1, m_{2}^{(\mu)}=2, m_{3}^{(\mu)}=3, \sigma_{1}^{(\mu)}=3, \sigma_{2}^{(\mu)}=2$, $\left.\sigma_{3}^{(\mu)}=1.\right)$

Using the polynomials $A_{n}$ introduced in Corollary 2.1, we denote by $\beta_{\nu}=\beta_{\nu}(X), \nu \geqq 1$,

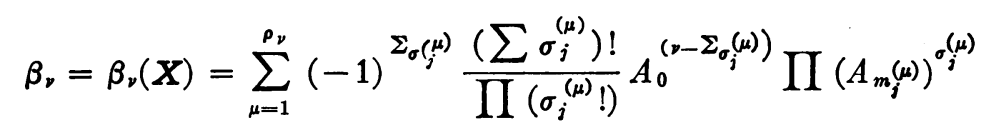

where $\sum \equiv \sum_{j=1}^{n_{\mu}}$, and $\Pi \equiv \prod_{m_{1}}^{n_{\mu}}$.

NotAtion. 2.4. $\alpha_{\nu}=\alpha_{\nu}(X), \nu \geqq 1$, will denote a polynomial built in the same way as the right-hand side of (2.15) where $A_{m_{j}}$ is replaced by $A_{2 N-m_{j}}$, and $A_{0}$ by $A_{2 N} . N$ is the highest power of $\zeta$ in $q\left(x+(i y / 2)\left(\zeta+\zeta^{-1}\right)+(z / 2)\left(\zeta-\zeta^{-1}\right), \zeta\right)$.

REMARK 2.5. The $\alpha_{\nu}$ and $\beta$, depend only upon the R-manifold $R$. Their degree in $x, y, z$ is $\nu n$. Here, $n$ is the degree of $q(u, \zeta)$ in $u$ (see Corollary 2.1). Further, $\alpha_{0} \equiv \beta_{0} \equiv 1$.

EXAMPLES. $\beta_{1}=-A_{1}, \quad \beta_{2}=A_{1}^{2}-A_{2} A_{0}, \quad \beta_{3}=-A_{1}^{3}+A_{1} A_{2} A_{0}-A_{3} A_{0}^{2}, \cdots$, $\alpha_{1}=-A_{2 N-1}, \alpha_{2}=A_{2 N-1}^{2}-A_{2 N-2} A_{2 N}, \cdots$

$\left.{ }^{(22}\right)$ As indicated in Corollary 2.1, the $A_{\nu}(X)$ are polynomials (2.10) with $m, M, b_{k s}$ replaced by $n, N$, and $a_{k s}$, respectively. 
LEMMA 2.3. Let $\zeta$ be a solution of the equation

$$
Q(\zeta ; X)=\sum_{\mu=0}^{2 N} A_{\mu} \zeta^{\mu}=0 .
$$

Then

$$
\zeta^{2 N+l}=-\sum_{\mu=0}^{2 N-1} \sum_{j=0}^{\min (l, \mu)} \alpha_{l-j} \frac{A_{\mu-j}}{A_{2 N}^{l-j+1}} \zeta^{\mu}, \quad l \geqq 0,
$$

and

$$
\zeta^{-1-l}=-\sum_{\mu=0}^{2 N-1} \sum_{j=0}^{\min (2 N-1-\mu, l)} \beta_{l-j}\left(A_{\mu+j+1} / A_{0}^{l-j+1}\right) \zeta^{\mu}, \quad l \geqq 0
$$

where $\alpha_{j}$ and $\beta_{j}$ are described in Notations 3 and 4 respectively.

REMARK 2.6. For every $A$, appearing in the right-hand side of (2.17) and (2.18) for which $\nu<0$, or $\nu>2 N$, we have to substitute 0 . The above formulas (2.9), (2.17), and (2.18) are obtained by formal computation.

In order to derive the formulas (2.13a) and (2.13b) we first apply Lemma 2.2. For any fixed value of $X=X^{(0)}$ for which no two roots $\zeta^{(\nu)}(X), \nu=1, \cdots$, $2 N$ coincide, we can evaluate

$$
\frac{1}{2 \pi i} \int_{\mathcal{L}} \frac{P(\zeta, X) \zeta^{N-M}}{Q(\zeta, X)} \frac{d \zeta}{\zeta}
$$

by the residue theorem: We choose the path of integration $\mathcal{L}$, so that inside $\mathcal{L}$, lies one and only one $\operatorname{root} \zeta^{(v)}(X), X$ belonging to a sufficiently small neighborhood of $\boldsymbol{X}^{(0)}$.

Now

$$
\begin{aligned}
P(\zeta, X)[\zeta Q(\zeta, X)]^{-1} & \\
= & {\left[\zeta^{N-M-1} \sum_{s=0}^{2 M} G_{s} \zeta^{s}\right] / A_{2 N}\left(\zeta^{2 N}+\sum_{\nu=0}^{2 N-1} A_{2 N}^{-1} A_{\nu} \zeta^{\nu}\right) \equiv S }
\end{aligned}
$$

by (2.9).

1. If $N>M$ we can evaluate $(2 \pi i)^{-1} \int \mathcal{C}, S d \zeta$ directly by the residue theorem, to obtain

$$
\left[\sum_{s=0}^{2 M} G_{s} \zeta^{s+N-M-1} / \frac{\partial Q}{\partial \zeta}\right]_{\zeta=\zeta}(\nu)
$$

Suitably changing the index of summation:

$$
\mu=s+N-M-1,
$$

we obtain (2.13a). 
2. If $N \leqq M$ we have three groups of terms in the numerator, depending on whether the exponent of $\zeta$ is negative, positive and less than $2 N$, or greater than $2 N-1$. If the first and the third alternatives occur, we employ (2.17) and (2.18) in order to change the exponents of $\zeta$, to lie between 0 and $2 N-1$. We then obtain

$$
\begin{aligned}
(2 \pi i)^{-1} \int_{\mathcal{L}_{\nu}} S d \zeta= & \left\{( \frac { \partial Q } { \partial \zeta } ) ^ { - 1 } \left[\sum_{s=0}^{M-N} G_{M-N-s} \zeta^{-1-s}\right.\right. \\
& \left.\left.+\sum_{s=0}^{2 N-1} G_{M-N+1+s} \zeta^{s}+\sum_{s=0}^{M-N-1} G_{M+N+1+s} \zeta^{2 N+s}\right]\right\}_{\zeta=\zeta}(\nu) \\
=\left\{\left(\frac{\partial Q}{\partial \zeta}\right)^{-1}\right. & {\left[\sum _ { \mu = 0 } ^ { 2 N - 1 } \left(G_{M-N+1+\mu}\right.\right.} \\
& -\sum_{s=0}^{M-N} \sum_{j=0}^{\min (2 N-1-\mu, s)} \beta_{s-j} A_{0}^{-s+j-1} A_{\mu+j+1} G_{M-N-s} \\
& \left.\left.\left.-\sum_{s=0}^{M-N-1} \sum_{j=0}^{\min (s, \mu)} \alpha_{s-j} A_{2 N}^{-s+j-1} A_{\mu-j} G_{M+N+s+1}\right) \zeta^{\mu}\right]\right\}_{\zeta=\zeta}^{(\nu)}
\end{aligned}
$$

by (2.17) and (2.18).

Collecting coefficients of $\zeta^{\mu}$ and writing the factor

$$
A_{0}^{-1}\left(A_{0} A_{2 N}\right)^{-M+N}(\partial Q / \partial \zeta)^{-1}
$$

before the bracket, we obtain in this case

$$
\begin{aligned}
L^{(\mu)}=A_{0}^{M-N+1} & A_{2 N}^{M-N} G_{M-N+1+\mu} \\
& -\sum_{s=0}^{M-N} \sum_{j=0}^{\min (2 N-1-\mu, s)} \beta_{8-j} A_{\mu+j+1} A_{0}^{M-N-s+j} A_{2 N}^{M-N} G_{M-N-s} \\
& -\sum_{s=0}^{M-N-1} \sum_{j=0}^{\min (s, \mu)} A_{0}^{M-N+1} A_{2 N}^{M-N-8+j-1} A_{\mu-j} \alpha_{s-j} G_{M+N+s+1 .}
\end{aligned}
$$

REMARK 2.7. We note that (in general) in this case $L^{(\mu)}$ has neither $A_{0}$ nor $A_{2 N}$ as a factor, so that the degree of $L^{(\mu)}$ cannot be decreased.

REMARK 2.8. By introducing $R^{-1} H\left(R^{-3} x^{\prime}, R^{-3} y^{\prime}, R^{-3} z^{\prime}\right), R=\left(x^{\prime 2}+y^{\prime 2}+z^{\prime 2}\right)^{1 / 2}$, we can study the behavior of $H$ at infinity.

3. The number of constants $b_{j, k}$ which appear in $L^{(\mu)}$.

Notation. 2.5. In the following, the sum

$$
[1 / 2]+[2 / 2]+\cdots+[x / 2]=\left(x^{2}+x+2[x / 2]\right) / 4
$$

will be denoted by $S(x)$.

Notation. 2.6. We shall further write 


$$
a(\nu)=\min (m,[(M-\nu-1) / 2]) .
$$

$a(\nu)$ depends upon $m$ and $M$, but since these quantities are supposed to be fixed in our discussion we do not indicate this dependence.

LeMma 2.4. Let $p(u, \zeta)$ be a polynomial of the form (2.7).

1. Then the expression $G_{s}$ given in (2.9) involves the $b_{j, \nu+j}$ where $\nu$ and $j$ range over

$$
\begin{aligned}
& (-M+\max (0, s-2 m)) \leqq \nu \leqq-M+s, \\
& {[(s-M-\nu) / 2] \leqq j \leqq a(\nu) .}
\end{aligned}
$$

2. The number of constants $b_{j, v+j}$ which appear in $G_{s}$ is

$$
m(s+1)+A(M-m)+\mathrm{S}(s-A)-\mathrm{S}(s)-\mathrm{S}(B) .
$$

Here:

$$
A=\min (0, s-2 M+2 m+1), \quad B=\min (2 m, s) .
$$

Proof. We note at first that in $G_{s}$, see (2.9), there appear two double sums of terms to which we shall refer as the first and second group of terms. Let us denote the number $\nu$ in $b_{j, v+j}$ as the index of $b_{j, v+j}$.

REMARK 2.8. We note that according to (2.7), the constants $b_{j, \nu+j}$ which exist for a given $\nu$ have $j$ ranging over $0 \leqq j \leqq a(\nu)$.

We assume for the time being that $s$ and $j$ are fixed and consider the range of variation of the index $\nu$. It assumes all integer values between two limits, which we now proceed to determine.

By inspecting (2.9) we see that the maximum index will always come when $\lambda=0$ in the first summation, and hence is equal to $-M+s$.

If $s$ is even, the minimum index will appear in the first group in (2.9) and will be equal to $-M+s-2 \min (s / 2, m)=-M+\max (0, s-2 m)$. If $s$ is odd and if $s \geqq 2 m+1$, then again the minimum index appears in the first group and is equal to $-M+s-2 m$. If $s<2 m+1$, then the minimum index appears in the second group and is equal to $-M$. Hence in all cases the minimum index is $-M+\max (0, s-2 m)$.

It remains now to determine the range of variation of $j$ for a given $s$ and $\nu$.

1. If $\nu=-M+s-2 \lambda$, then $\lambda=(-M+s-\nu) / 2$ and hence $j$ varies from $(-M+s-\nu) / 2$ to $a(\nu)$.

2. If $\nu=-M+s-2 \lambda+1$ we obtain $j_{\min }=[(-M+s-\nu) / 2]$ and $j_{\max }$ as above. This completes the proof of the first part of the lemma.

Using the above result we then determine the number of constants (see (2.29)) appearing in each $G_{8}$.

Lemma 2.5. Each $G_{s}$ is (in general) of degree $m$ in $x, y, z$.

Proof. For a given $s$, set $\lambda_{s}=\max (0,[s / 2]-(M-m))$ and $j_{s}=m$. Then set $b_{j_{s},-M+j_{s}+s-2 \lambda_{s}}=1$, and all other $b_{j, \kappa}=0$. Then $G_{s}=\Gamma_{j_{s}, 2 \lambda_{s}}=\Gamma_{m, 2 \lambda_{s}}$; hence by 
Remark 2.2, $G_{s}$ is, in general, of degree $m$.

In the same manner we obtain the following corollary.

Corollary 2.2. The coefficients $A$, in (2.11) are of the degree $n$.

Theorem 2.2. If $M<N$ the degree of $G_{\mu-M+N+1}$ (see (2.13a)) in $x, y$, and $z$ is $m$; if $M \geqq N$ the degree of $L^{(\mu)}$ is (see (2.13b))

$$
n(2 M-2 N+1)+m \text {. }
$$

Proof. (1) The above statement in the first case follows immediately from Remark 2.2 and Lemma 2.5.

(2) In the second case, $L^{(\mu)}$ is given by (2.25). We have three groups of terms and we have to inspect their degrees.

By Remark 2.5 and Corollary 2.2, it follows that the degree of the first and second groups are

$$
\begin{gathered}
n(2 M-2 N+1)+m, \\
n(s-j)+n+n(M-N-s+j)+n(M-N)+m=n(2 M-2 N+1)+m,
\end{gathered}
$$

respectively, and the third group also has the same degree.

TheOREM 2.3. 1. If $M \geqq N$, then all $L^{(\mu)}$ have in common the constants $b_{j, v+j}$ where $\nu$ and $j$ range over:

$$
-M \leqq \nu \leqq-N, \quad 0 \leqq j \leqq a(\nu)
$$

and

$$
\begin{aligned}
& \max (-N+1, N+1-2 m) \leqq \nu \leqq M \\
& \max (0,[(N+1-\nu) / 2]) \leqq j \leqq a(\nu) .
\end{aligned}
$$

In addition each $L^{(\mu)}$ involves $b_{j,-N+1+j+\sigma}$ where

$$
0 \leqq \sigma \leqq \mu, \quad[(\mu-\sigma) / 2] \leqq j \leqq[(2 N-\sigma) / 2]-1, \quad \text { if } N \leqq m,
$$

and

$$
\begin{aligned}
& \max (0, \mu-2 m) \leqq \sigma \leqq \mu \\
& {[(\mu-\sigma) / 2] \leqq j \leqq \min (m,[(2 N-\sigma) / 2]-1) \quad \text { if } \quad N>m .}
\end{aligned}
$$

2. Let $\tau=\max (m, N)$. Then all $L^{(\mu)}$ involve

$$
(2 M-2 N+1)(m+1)-(\tau-N)^{2}
$$

common constants $b_{j, \kappa}$ and each individual $L^{(\mu)}$ involves, in addition,

$$
\frac{(\mu+1)(2 N-\mu)}{2}
$$

constants if $N \leqq m$ and 


$$
(B+1)(m+1)+\mathrm{S}(A)-\mathrm{S}(B)+\left[\frac{A-1}{2}\right]
$$

if $N>m$. Here $A=\min (0,2 N-2 m-\mu-2) ; B=\min (\mu, 2 m)$.

Proof. 1. By inspecting (2.25) we see that for $M \geqq N$ the subscripts $s$ of $G_{8}$ appearing in an $L^{(\mu)}$ range over the intervals:

$$
\text { (1) }\{0, M-N\}, \text { (2) }\{M-N+1+\mu\} \text {, }
$$

(3) $\{M+N+1,2 M\}$.

(The interval (2) consists of one term only.) The sets (1) and (3) are common to all $L^{(\mu)}$, and we determine at first the $b_{j, \nu+j}$ which appear in both intervals.

Let us determine at first the values of the indices $\nu$ of those $b_{j, \nu+j}$ which appear in $G_{s}$ but not in $G_{s-1}$. By Lemma 2.4, we see that only one value of $\nu$, namely $\nu=-M+s$, belongs to this category. According to (2.28) the range of $j$ for this value of $\nu$ is

$$
0 \leqq j \leqq a(\nu) .
$$

Further, from (2.28) we see that for the smallest $s, s=0$, there appears only one value of $\nu, \nu=-M$. Hence in the first group will appear the $b_{j, v+j}$, with $(\nu, j)$ indicated in (2.31).

In considering the group (3) we have to take into account that the $b_{j, \nu+j}$ which appear in the first term $G_{M+N+1}$ are, according to (2.28),

$$
\begin{array}{r}
-M+\max (0, M+N+1-2 m) \leqq \nu \leqq N+1, \\
{[(N+1-\nu) / 2] \leqq j \leqq a(\nu) .}
\end{array}
$$

We determine as before the $b_{j, v+j}$ appearing in the remaining $G_{\boldsymbol{s}}$ belonging to the third group to obtain instead of (2.39)

$$
\begin{aligned}
&-M+\max (-M, N+1-2 m) \leqq \nu \leqq M ; \\
& \max (0,[(N+1-\nu) / 2]) \leqq j \leqq a(\nu) .
\end{aligned}
$$

Eliminating from (2.41) what appeared already in (2.31), we obtain $(\nu, j)$ indicated in (2.32).

We have finally to determine the $(\nu, j)$ of those $b_{j, v+j}$ which appear in $L^{(\mu)}$ but not in (2.31) nor in (2.32). These $b_{j, v+j}$ are the constants appearing in $G_{M-N+\mu+1}$, see (2.38), but not included in (2.31) and (2.32).

By Lemma 2.4, $G_{M-N+\mu+1}$ involves the constants $b_{j, \nu+j}$ for

$$
\begin{aligned}
& \max (-M,-N+1+\mu-2 m) \leqq \nu \leqq-N+1+\mu \\
& {[(-N+1+\mu-\nu) / 2] \leqq j \leqq a(\nu) }
\end{aligned}
$$

and it remains only to eliminate those $b_{j, v+j}$ which have been already counted. We have to distinguish two cases.

1. $N \leqq m$. In this case in (2.32) $\max (-N+1, N+1-2 m)=-N+1$. According to Remark 2.8, (2.31) includes all $b_{j, y+j}$, with index $\nu$. Hence we can 
replace for $\nu \leqq-N$, the inequality for $\nu$ in (2.42) by

$$
-N+1 \leqq \nu \leqq-N+\mu+1 .
$$

Let us write for the moment $\sigma=\nu+N-1$. Then (2.43) becomes

$$
0 \leqq \sigma \leqq \mu \text {. }
$$

According to the second inequality in (2.42) the lower bound for $j$ becomes

$$
[(\mu-\sigma) / 2]
$$

while according to $(2.32)$ in all $L^{(\mu)}$ the lower bound for $j$ is $[(2 N-\sigma) / 2]$. Hence, the $(j, \sigma)$ of additional constants $b_{j,-N+1+\sigma+j}$ in $L^{(\mu)}$ lie in the range (2.33). A similar discussion yields (2.34).

2. We proceed now to the determination of the number of constants $b_{j, \nu+j}$ in (2.31) and (2.32) as well as in (2.33) and (2.34).

Obviously, for every $\nu$ the number of constants is one more than the difference between upper $\left(l_{\nu}^{+}\right)$and lower $\left(l_{\nu}^{-}\right)$limits of $j$ in the second inequalities of (2.31), (2.32), (2.33), (2.34), respectively. In order to obtain (2.35) we subtract from the sum $\sum_{\nu} l_{\nu}^{+}$of all upper limits the sum $\sum_{\nu} l_{\nu}^{-}$of all lower limits and add the number of $\nu$ involved.

We have to consider two cases.

1. $m \geqq N$. For $-M \leqq \nu \leqq M-2 m, l_{\nu}^{+}=m$, and for the remaining $\nu, l_{\nu}^{+}$ $=[(M-\nu-1) / 2]$. Hence $\sum_{\nu} l_{\nu}^{+}=m(2 M-2 m+1)+\mathrm{S}(2 m-2) . \sum_{\nu} l_{\nu}^{-}$is determined as follows:

For $-M \leqq \nu \leqq-N$, we have $l_{\nu}^{-}=0$; for $-N+1 \leqq \nu \leqq N+1$, we have $l_{\nu}^{-}=[(N+1-\nu) / 2]$ and for the remaining $\nu, l_{\nu}^{-}=0$.

Hence $\sum_{\nu} l_{\nu}^{-}=\mathrm{S}(2 N)$. Finally we note that the number of $\nu$ is $2 M+1$. Thus in this case we have $((2 M-2 m+1) m+\mathrm{S}(2 m-2)-\mathrm{S}(2 N)+2 M+1)$ constants. This can be written in the form (2.35) with $\tau=m$.

2. $m<N$. Then $\max (-N+1, N+1-2 m)=N+1-2 m$. For $-M \leqq \nu$ $\leqq-N, l_{\nu}^{+}=m, l_{\nu}^{-}=0$, and hence the number of constants is $(M-N+1)(m+1)$. For $N+1-2 m \leqq \nu \leqq M-2 m, \quad l_{\nu}^{+}=m$, and for $N-2 m+1 \leqq \nu \leqq M, \quad l_{\nu}^{+}$ $=[(M-\nu-1) / 2]$. For $N+1-2 m \leqq \nu \leqq N+1, l_{\nu}^{-}=[(N+1-\nu) / 2]$, and for the remaining terms, $l_{\nu}^{-}=0$. The number of terms is $M-N+2 m$. Hence the total number of constants is

$$
\begin{aligned}
(M-N+1)(m+1)+(M-N) m+\mathrm{S}(2 m-2) & -\mathrm{S}(2 m)+(M-N+2 m) \\
& =(2 M-2 N+1)(n+1) .
\end{aligned}
$$

We now consider the number of additional constants in each $L^{(\mu)}$. For $0 \leqq \sigma \leqq \mu, l_{\sigma}^{+}=[(2 N-\sigma) / 2]-1$ and $l_{\sigma}^{-}=[(\mu-\sigma) / 2]$. Hence $\sum_{\sigma} l_{\sigma}^{+}=\mathrm{S}(2 N)$ $-\mathrm{S}(2 N-\mu-1)-(\mu+1), \sum_{\sigma} l_{\sigma}^{-}=\mathrm{S}(\mu)$, and therefore the number of constants is $\mathrm{S}(2 N)-\mathrm{S}(2 N-\mu-1)-\mathrm{S}(\mu)$. Now, by an easy calculation

$$
\mathrm{S}(A)+\mathrm{S}(B)=\mathrm{S}(A+B)-[A B / 2] \text {. }
$$


Applying this and (2.26) we see that the number of constants is (2.35). In a similar manner the formula (2.37) is derived.

TheOREM $2.4\left({ }^{23}\right)$. Let $M \geqq N$, and $k>2 N+4 n+4$. If the $L^{(\mu)}$ are of the degree not higher than $k$ in $x, y, z$, then the maximum number of constants $b_{j, k}$ which can appear simultaneously in every $L^{(\mu)}$ is

$$
\sigma(k-n \sigma+1)-(k-n \sigma-N)^{2} .
$$

In addition, in this case, each individual $L^{(\mu)}$ involves

$$
(\mu+1)(2 N-\mu) / 2
$$

constants $b_{j, \kappa}$ which vary from term to term $\left({ }^{24}\right)$. Here $\sigma$ has to be determined as follows.

Let $\sigma_{\kappa}$, and $\tau_{\kappa}, \kappa=1,2$, be given by the relations

$$
\begin{aligned}
\sigma_{1}(n+1)+\tau_{1} & =k-N, & & \text { where } 0 \leqq \tau_{1} \leqq n, \\
2 \sigma_{2}\left(n^{2}+n\right)+\tau_{2} & =k, & & \text { where } 0 \leqq \tau_{2} \leqq 2 n(n+1)-1,
\end{aligned}
$$

and $\phi=\left(2 \tau_{1} n+\tau_{2}+1\right) /(4 n(n+1)), j=2\left[\left(2 \sigma_{1}+2 \sigma_{2}+2 \phi+1\right) / 4\right]-\left(\sigma_{1}+\sigma_{2}+1\right)$.

Then $\left({ }^{25}\right)$

$$
\sigma=\sigma_{1}+\sigma_{2}+j .
$$

Proof. We assume at first that $k$ is sufficiently large, so that the maximum value of (2.35) is assumed for a value $m \geqq N$, so that we can write $\tau=m$. (The lower bound for $k$ will be determined later.)

We write $x$ in (2.35) instead of $m, y$ instead of $M$, and consider

$$
(2 y-2 N+1)(x+1)-(x-N)^{2}
$$

and we assume for the time being that $x$ and $y$ vary continuously over all real positive values. According to (2.30) $x$ and $y$ are connected by the relation

$$
k=x+2 n y+n-2 N n \text {. }
$$

Using (2.50) we can write (2.49) either as a function, say $f(x)$, of $x$ alone, or as a function, say $g(y)$, of $y$ alone. $f$ and $g$ are both quadratic in $x$ and $y$, respectively.

The solution of $f^{\prime}(x)=0$ is

$$
x_{\max }=\frac{N n+k / 2-1 / 2}{n+1} .
$$

${ }^{(23)}$ This theorem can be considered as an analogue of the Riemann-Roch theorem applied to functions possessing poles only at infinity.

${ }^{(24)}$ The constants mentioned in the second group can appear in several $L^{(\mu)}$ but not in all.

(25) We note that if $\sigma_{1}+\sigma_{2}$ is even, then $j=1$; if $\sigma_{1}+\sigma_{2}$ is odd, then $j=0$ for $\phi \leqq 1 / 2$ and $j=2$ for $\phi>1 / 2$. 
According to (2.50), the corresponding $y$ is

$$
\begin{aligned}
y_{\max } & =\left(N-\frac{1}{2}+\frac{k}{2 n}\right)\left(\frac{n+1 / 2}{n+1}\right)-\frac{n-1}{4 n(n+1)} & \\
& =N+\frac{\sigma_{1}+\sigma_{2}-1}{2}+\phi, & 0<\phi<1 .
\end{aligned}
$$

Since the function $g(y)$ is quadratic and therefore is symmetric about the line $y=y_{\max }$, the integer nearest to $y_{\max }$ is

$$
M_{\max }=N+\frac{\sigma_{1}+\sigma_{2}+j-1}{2},
$$

where $j$ is defined as above. The corresponding $x_{0}$ is

$$
x_{0}=k-n\left(\sigma_{1}+\sigma_{2}+j\right),
$$

and the number of constants is

$$
\sigma(k-n \sigma+1)-(k-n \sigma-N)^{2} .
$$

We proceed now to the determination of the lower bound for $k$.

The number of constants which appear in $L^{(\mu)}$ is given by

$$
\begin{array}{ll}
\rho_{1}=\frac{1}{n}(k-m)(m+1) & \text { for } m \leqq N, \\
\rho_{2}=\frac{1}{n}(k-m)(m+1)-(m-N)^{2} & \text { for } m \geqq N .
\end{array}
$$

Let

$$
\rho=\left\{\begin{array}{l}
\rho_{1} \text { for } m \leqq N \\
\rho_{2} \text { for } m \geqq N .
\end{array}\right.
$$

This formula is obtained by substituting (2.50) with $x$ replaced by $m$ and $y$ replaced by $M$ into (2.35).

Assuming for the moment that $m=x$ varies continuously so that $\rho_{1}(x)$, $\rho_{2}(x), \rho(x)$ are defined in the whole interval $0 \leqq x<\infty$ and not only for integer values, both curves

$$
y=\rho_{1}(x), \quad y=\rho_{2}(x)
$$

are concave parabolas; and if at the point of their intersection $\left({ }^{26}\right) m=N$,

$$
\text { (a) } \rho_{1}^{\prime}(x)_{x=N}>0, \quad \text { (b) } \rho_{2}^{\prime}(x)_{x=N}>0 \text {, }
$$

then the maximum of $\rho(x)$ will take place in the interval $m \geqq N$.

If now we return to the variable $m$ which assumes only discrete values

(20) This requirement leads us to a condition on $k$. 
and, in addition, take into account that $M$ must be an integer, $M \geqq N$, then we see that the condition (b) has to be replaced by a condition which takes into account these additional requirements. Indeed since $m$ and $M$ assume only integer values it could occur that despite the fulfillment of conditions (a) and (b), $m_{\max }<x_{\max }$ and will lie in the part $m<N$.

From (2.50) we see that if $M$ is an integer, $m$ is also an integer while the reverse is not always true. It is therefore convenient to operate with $M$ rather than with $m$.

$y\left(x_{\max }\right)$ is given in $(2.52)$ and we shall at first require that the integer $M_{\max }$ nearest to $y\left(x_{\max }\right)$ is not smaller than $N$. Then, since from the definition of $\sigma_{1}$ and $\sigma_{2}$ we have

$$
\sigma_{1}>\frac{k-N}{n+1}-1 ; \quad \sigma_{2}>\frac{k}{2\left(n^{2}+n\right)}-1,
$$

it follows that

$$
\frac{k-N}{n+1}+\frac{k}{2\left(n^{2}+n\right)}>3
$$

which is certainly satisfied if $k>N+3 n+3$.

Finally, we wish that the $m_{\max }$ corresponding to $M_{\max }$ is also $\geqq N$. By (2.54), $m_{\max }=k-n\left(\sigma_{1}+\sigma_{2}+j\right)$. Now by the definition of $\sigma_{1}, \sigma_{2}$, and $j$, we have

$$
\sigma_{1} \leqq \frac{k-N}{n+1}, \quad \sigma_{2} \leqq \frac{k}{2 n(n+1)}, \quad \text { and } \quad j \leqq 2 .
$$

Therefore

$$
m_{\max } \geqq k-n\left(\frac{k-N}{n+1}+\frac{k}{2 n(n+1)}+2\right)=\frac{N n+k / 2-2 n-2}{n+1},
$$

which is certainly not greater than $N$ if $k>2 N+4 n+4$. We see that the last is the most restrictive condition, and hence $k>2 N+4 n+4$ is our final bound, which is a sufficient condition in order that (2.47) be the number of constants which appear simultaneously in all $L^{(\mu)}$. According to Theorem 2.3, in each individual $L^{(\mu)}$ appear the number of constants given by (2.36), which is independent of $k$.

4. A class of harmonic functions with algebraic associates and their properties. One of the aims of our investigation is to study harmonic functions with algebraic $\mathrm{C}_{3}$-associates. As we stressed in $\$ I I .1$, such harmonic functions are not necessarily algebraic, and one of the problems which arises is to determine those associates which generate algebraic harmonic functions. A problem which is closely related to the above-mentioned question is that of finding sets of $\mathrm{C}_{\mathbf{3}}$-associates possessing the property that certain linear combinations of the corresponding harmonic functions are algebraic. 
The $\mathrm{C}_{3}$-associates of functions considered in $\$ I I .2$ are of the form

$$
\sum_{\mu=0}^{2 N-1} \frac{S^{(\mu)}\left(Z, Z^{*}\right) \zeta^{\mu}}{\partial\left(\zeta^{N} q\left[\left(Z^{1 / 2} \zeta^{1 / 2}+Z^{* 1 / 2} \zeta^{-1 / 2}\right)^{2}, \zeta\right]\right) / \partial \zeta}
$$

where $\zeta$ is a solution of the equation

$$
q\left[\left(Z^{1 / 2} \zeta^{1 / 2}+Z^{* 1 / 2} \zeta^{-1 / 2}\right)^{2}, \zeta\right]=0 .
$$

On the other hand $S^{(\mu)}\left(Z, Z^{*}\right)$ have a special form. It is of interest, in connection with the second problem mentioned above, to consider a somewhat larger class of algebraic associates, namely functions

$$
H_{n, k, a}^{(1)}(X), \quad H_{n, k, a, 8}^{(2)}(X), \quad H_{n, k, a, 8}^{(8)}(X),
$$

whose $\mathrm{C}_{3}$-associates are

$$
\begin{aligned}
D_{n, k, a}^{(1)} & =\frac{Z^{(n+k) / 2} Z^{*(n-k) / 2} \zeta^{a}}{\partial\left[\zeta^{N} q\left[\left(Z^{1 / 2} \zeta^{1 / 2}+Z^{* 1 / 2} \zeta^{-1 / 2}\right)^{2}, \zeta\right]\right] / \partial \zeta}, \\
D_{n, k, a, 8}^{(2)}\left(Z, Z^{*}\right) & =\frac{Z^{(n+k) / 2} Z^{*(n-k) / 2} \zeta^{a}}{A_{0}^{g} \partial\left[\zeta^{N} q\left[\left(Z^{1 / 2} \zeta^{1 / 2}+Z^{* 1 / 2} \zeta^{-1 / 2}\right)^{2}, \zeta\right]\right] / \partial \zeta} \\
D_{n, k, a, 8}^{(3)}\left(Z, Z^{*}\right) & =\frac{Z^{(n+k) / 2} Z^{*(n-k) / 2} \zeta^{a}}{A_{2 N}^{g} \partial\left[\zeta^{N} q\left[\left(Z^{1 / 2} \zeta^{1 / 2}+Z^{* 1 / 2} \zeta^{-1 / 2}\right)^{2}, \zeta\right]\right] / \partial \zeta}
\end{aligned}
$$

respectively; where $\zeta$ satisfies equation (2.59) and where $n, k, a, s, n \geqq 0$, $0 \leqq a \leqq 2 N-1, s>0$, are integers. $H_{n, k, a, s}^{(1)}(X), H_{n, k, a, s}^{(2)}(X)$, and $H_{n, k, a, s}^{(3)}(X)$ are in general not algebraic functions. On the other hand, we shall show that certain linear combinations of the above functions are algebraic.

In order to formulate our results it is useful to introduce at first some notations:

In (2.11), (2.15), and in the line following (2.15), the quantities $A_{\nu}, \alpha_{\nu}, \beta_{\nu}$ (depending only on the $\mathrm{R}$-manifold $R$ ) have been introduced. They are combinations of $\Gamma_{\nu, \lambda}$ introduced in (2.5). In the characteristic space $\mathcal{C}$, we have $\Gamma_{\nu, \lambda}=C_{2 v, \lambda} Z^{\lambda / 2} Z^{* \nu-\lambda / 2}$, see (2.6). Therefore the expressions

$$
-\sum_{j=0}^{\min (l, \mu)} \alpha_{l-j} A_{\mu-j} A_{2 N}^{j} \text { and }-\sum_{j=0}^{\min (2 N-1-\mu, l)} \beta_{l-j} A_{\mu+j+1} A_{0}^{j}
$$

can be written as functions of $Z$ and $Z^{*}$, namely as

$$
\sum_{a, b} \gamma_{a, b}^{(l, \mu)} Z^{a} Z^{* b} \text { and } \sum_{a, b} \delta_{a, b}^{(l, \mu)} Z^{a} Z^{* b}
$$

respectively. $\gamma_{a, b}^{(l, \mu)}$ and $\delta_{a, b}^{(l, \mu)}$ are numerical constants. Further we denote as before by $2 N$ the degree of $Q(\zeta, X)$, see (2.10), considered as a function of $\zeta$. Obviously all those quantities depend only on $R$. 
THEOREM 2.5. Let $s$ and $\nu, \nu \geqq 0$, be two integers. Then the expressions

$$
\sum_{\lambda=0}^{2 \nu} C_{2 v, \lambda} d_{8}\left(D_{\nu, \lambda, 8-\nu+N+\lambda-1}^{(1)}\right), \quad \text { for }-N+\nu \leqq s \leqq N-\nu,
$$

$$
\begin{aligned}
& \sum_{\mu=8-\nu+N-1}^{2 N-1} C_{2 \nu, \mu-8+\nu-N+1} \mathrm{~d}_{3}\left(D_{\nu, \mu-8-N+1, \mu}^{(1)}\right) \\
& +\sum_{j=0}^{s+\nu-N+1} \sum_{\mu=0}^{2 N-1} \sum_{a, b} C_{2 \nu, N+\nu-8+j+1} \gamma_{a, b}^{(j, \mu)} \mathrm{d}_{3}\left(D_{\nu+a+b, N-s+1+j+a-b, \mu, j}^{(2)}\right) \\
& \quad \text { for } N-\nu<s \leqq N+\nu,
\end{aligned}
$$

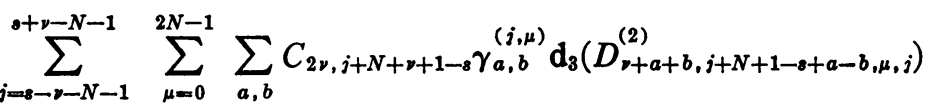

$$
\begin{gathered}
\sum_{\mu=0}^{\nu+s} C_{2 \nu, \mu+\nu-s} \mathrm{~d}_{8}\left(D_{\nu, \mu-s, \mu}^{(1)}\right) \\
+\sum_{j=0}^{\nu-s-1} \sum_{\mu=0}^{2 N-1} \sum_{a, b} C_{2 \nu, \nu-s-j-1} \delta_{a, b}^{(j, \mu)} \mathrm{d}_{3}\left(D_{\nu+a+b,-s-j-1+a-b, \mu, j}^{(3)}\right) \\
\text { for }-N+1-\nu \leqq s \leqq-N+\nu . \\
\sum_{j=-\nu-s-N}^{\nu-s-N} \sum_{\mu=0}^{2 N-1} \sum_{a, b} C_{2 \nu, \nu-s-j-N} \delta_{a, b}^{(j, \mu)} \mathrm{d}_{8}\left(D_{\nu+a+b,-s-j-N+a-b, \mu, j}^{(3)} \quad \text { for } s \leqq-N-\nu\right.
\end{gathered}
$$

are algebraic harmonic functions of $x, y, z$ which are single-valued on the $\mathrm{R}$-manifold $R$, given by $(2.14)\left({ }^{27}\right)$.

Proof. If we choose in (2.7)

$$
p(u, \zeta)=u^{\nu} \zeta^{s}
$$

then according to Theorem 2.1, $\mathrm{B}_{3}(p / q)$ (see 1.15) is an algebraic function. If further $\nu$ and $s$ assume all integer values such that $\nu \geqq 0$, then $B_{3}\left(u^{\nu} \zeta^{s} / q\right)$ will yield the totality of linearly independent algebraic functions which are singlevalued on $R$, and can be obtained by our procedure. According to (1.12), (1.13), we have

$$
\begin{array}{r}
\frac{1}{2 \pi i} \int_{\mathcal{L}_{\mu} \frac{\left(Z^{1 / 2} \zeta^{1 / 2}+Z^{* 1 / 2} \zeta^{-1 / 2}\right)^{2 \nu} \zeta^{\circ}}{q\left[\left(Z^{1 / 2} \zeta^{1 / 2}+Z^{* 1 / 2} \zeta^{-1 / 2}\right)^{2}, \zeta\right]} \frac{d \zeta}{\zeta}} \\
=\sum_{\lambda=0}^{2 \nu} \frac{C_{2 \nu, \lambda} Z^{\lambda / 2} Z^{* \nu-\lambda / 2}\left(\zeta^{(\mu)}\right)^{-\nu+N+\lambda-1}}{\partial\left[\zeta^{(\mu)^{N}} q\left(\left(Z^{1 / 2} \zeta^{(\mu)^{1 / 2}}+Z^{* 1 / 2} \zeta^{(\mu)^{-1 / 2}}\right)^{2}, \zeta^{(\mu)}\right] / \partial \zeta^{(\mu)}\right.}
\end{array}
$$

(27) The operator $d_{3}$ was introduced on $p$. 465 . This operator is given by the right-hand side of (1.23), where, however, $x$ is not to be considered as derived from $H$, but as an independent function. 
where $\zeta^{(\mu)}$ is a solution of equation (2.59). If $-N+\nu<s \leqq N-\nu$, then the powers $\kappa\left(\right.$ of $\left.\zeta^{(\mu)}\right)$ lie in the range $[0 \leqq \kappa \leqq 2 N-1]$. Since $\mathbf{B}_{3}\left(u^{\nu} \zeta^{\%} / q\right)$ is an algebraic harmonic function, it follows directly that (2.64) is an algebraic expression in $x, y, z$. If on the other hand, $s>N-\nu$ or $s \leqq-N+\nu$, then in the right-hand side of (2.70) there appear powers of $\zeta^{(\mu)}$ lying outside $[0,2 N-1]$ which must be reduced to the powers lying in the above range. This is done by applying (2.17) and (2.18). If we then use (2.62) and (2.63) and separate the cases as to whether all or not all terms have to be reduced, we get (2.65)(2.68).

\section{HARMONIC VECTORS}

1. Preliminaries. In addition to the harmonic functions it is of interest to consider harmonic vectors $H$. These vectors possess many features similar to those of analytic functions and have been considered previously in different connections. In particular, we wish to mention that von Mises [12] and others have indicated that a generalized Cauchy formula holds for them.

The importance of introducing an associate $f(u, \zeta)$ to a harmonic function is in part based on the fact that $f(u, \zeta)$ can be considered as associate of the vector $H=\left(H_{1}, H_{2}, H_{3}\right)$, where $f$ is the associate in the previous sense of the component $H_{1}$ considered as a harmonic function. However, $\mathrm{H}_{2}$ and $\mathrm{H}_{3}$ are not uniquely determined. In fact, we have the following lemma.

Lemma 3.1. Let $H_{2}^{(0)}, H_{3}^{(0)}$ be functions such that $H=\left(H_{1}, H_{2}^{(0)}, H_{3}^{(0)}\right)$ forms a harmonic vector in $\mathrm{N}(0)$. Then

$$
\left\{H_{1}, H_{2}^{(0)}+\operatorname{Re}[g(y+i z)], H_{3}^{(0)}+\operatorname{Im}[g(y+i z)]\right\}
$$

where $g$ is an arbitrary analytic function of $(y+i z)$ which is regular in the projection of the domain $B$ on the $y, z$-plane forms the most general harmonic vector whose first component is $H_{1}$.

Thus

$$
\begin{aligned}
H= & \mathbf{R}_{3}\left(f, \mathcal{L}, X_{0}, g\right) \\
\mathbf{R}_{3}\left(f, \mathcal{L}, \boldsymbol{X}_{0}, g\right) \equiv & \left\{\mathbf{B}_{3}\left(f, \mathcal{L}, X_{0}\right), \mathbf{B}_{3}\left[\frac{i}{2}\left(\zeta+\zeta^{-1}\right) f(u, \zeta), \mathcal{L}, X_{0}\right]\right. \\
+ & \operatorname{Re}[g(y+i z)] \\
& \left.\mathbf{B}_{3}\left[\frac{1}{2}\left(\zeta-\zeta^{-1}\right) f(u, \zeta), \mathcal{L}, X_{0}+\operatorname{Im}[g(y+i z)]\right]\right\}
\end{aligned}
$$

generates harmonic vectors. (In this section, we omit the superscripts indicating the dimension, and write, for example, $\mathscr{B}$ and $\mathscr{B}^{\prime}$ instead of $\mathscr{B}^{3}$ and $\mathscr{B}^{\prime 2}$ respectively.)

Obviously there is a parallelism between the operations performed on 
harmonic vectors and those performed on the corresponding associates. In particular if we form $\int H_{1} d x+H_{2} d y+H_{3} d z$ this corresponds to forming the integrals $\int f(u) d u, \int g(\eta) d \eta$. This fact forms one basis of the subsequent considerations.

2. Harmonic vectors in the large. In the formula (3.2), $f(u, \zeta)$ and $g(\eta)$ are arbitrary analytic functions with the following domains of regularity: $f(u, \zeta)$ is regular for $\zeta$ on and interior to $\mathcal{L}$, and $u \in \mathcal{M}\left(X_{0}, \mathcal{L}\right)$ defined as the domain in which $u=x+i y\left(\zeta+\zeta^{-1}\right) / 2+z\left(\zeta-\zeta^{-1}\right) / 2$ varies as $\zeta$ varies on and interior to $\mathcal{L}$ and $\boldsymbol{X}$ in a sufficiently small neighborhood $U\left(X_{0}\right)$ of $\boldsymbol{X}_{0} . g(\eta)$ is regular in the projection of $U\left(\boldsymbol{X}_{0}\right)$ onto the $y+i z$ plane.

As indicated above, harmonic vectors behave in many respects in ways similar to analytic functions of a complex variable. There are, however, some important differences. If a harmonic function, say $H_{1}$, is given, then the remaining two components are determined within certain additive functions. Further, if $H_{1}$ is regular in a certain domain, say $\mathcal{B}$, then $H_{2}$ and $H_{3}$ are not necessarily regular there. In Lemma 3.2 , we give sufficient conditions that $\mathrm{H}_{2}$ and $\mathrm{H}_{3}$ exist.

LEMMA 3.2. Let $\mathbb{B}$ be a domain whose boundary is sufficiently smooth and which has the property that the intersection of every line parallel to the $x$-axis with $\mathbb{B}$ is (if not empty) a single (connected) segment (that is, its boundary consists of two points). Then to every harmonic function $H_{1}$ which is regular in the (closed) domain $B$ there exists a pair of functions $\mathrm{H}_{2}$ and $\mathrm{H}_{3}$ which are regular in $\mathcal{B}$ so that $H=\left(H_{1}, H_{2}, H_{3}\right)$ forms a harmonic vector.

Proof. According to the classical results, every harmonic function $H_{1}$ can be represented in the form

$$
\begin{aligned}
H_{1}(X) & =\iint_{\mathcal{B}^{\prime}} \frac{F(T) d \omega}{r}, \quad T=\left(t_{1}, t_{2}, t_{3}\right), \\
r & =\left[\left(x-t_{1}\right)^{2}+\left(y-t_{2}\right)^{2}+\left(z-t_{3}\right)^{2}\right]^{1 / 2},
\end{aligned}
$$

where $F$ is suitably chosen continuous function defined on the boundary $\mathcal{B}^{\prime}$ of B.

From the hypotheses made on $\mathcal{B}$, it follows that its boundary $\mathscr{B}^{\prime}$ can be subdivided into two parts, $\mathcal{B}^{\prime}=\mathscr{B}_{1}^{\prime}+\mathcal{B}_{2}^{\prime}$ such that if $\left(t_{1}, t_{2}, t_{3}\right)$ is a point of $\mathcal{B}_{k}^{\prime}$, $k=1,2$, then the segment $(-1)^{k}\left(x-t_{1}\right)<0, y=t_{2}, z=t_{3}$ lies outside of $\mathcal{B}$ and that the boundary manifold separating $\mathscr{B}_{1}^{\prime}$ and $\mathcal{B}_{2}^{\prime}$ has a (two-dimensional) vanishing area.

(3.3) may be written

$$
H_{1}(\boldsymbol{X})=\iint_{\mathcal{B}_{1}^{\prime}} \frac{F(\boldsymbol{T})}{r} d \omega+\iint_{\mathcal{B}_{2}^{\prime}} \frac{F(\boldsymbol{T})}{r} d \omega .
$$

Now, $\left[r^{-1}, h_{2}^{(k)}, h_{3}^{(k)}\right]$ where 


$$
\begin{aligned}
h_{2}^{(k)}=h_{2}^{(k)}(X, T) & =(-1)^{k} \frac{\left(y-t_{2}\right)}{r\left[r+(-1)^{k}\left(x-t_{1}\right)\right]}, \\
h_{3}^{(k)} & =(-1)^{k} \frac{\left(z-t_{3}\right)}{r\left[r+(-1)^{k}\left(x-t_{1}\right)\right]}
\end{aligned}
$$

is a harmonic vector. The functions $h_{2}^{(\mathbf{k})}, h_{3}^{(\boldsymbol{k})}$ for $\left(t_{1}, t_{2}, t_{3}\right) \in \mathcal{B}_{k^{\prime}}$ are regular in $\mathcal{B}$. If, therefore, we determine

$$
H_{s}^{(0)}(\boldsymbol{X})=\sum_{k=1}^{2} \iint_{\mathcal{B}_{k}{ }^{\prime}} F(T) h_{s}^{(k)}(\boldsymbol{X}, \boldsymbol{T}) d \omega, \quad s=2,3,
$$

then $H_{s}^{(0)}(X)$ will be regular harmonic functions in $\mathbb{B}$ and $\boldsymbol{H}=\left(H_{1}, H_{2}^{(0)}, H_{3}^{(0)}\right)$ will form a harmonic vector in $\mathbb{B}$.

On the other hand, if $B$ is not a domain of the type described in Lemma 3.2 , then to a given harmonic function there does not necessarily exist a pair of harmonic functions $H_{2}, H_{3}$ which are regular in $\mathcal{B}$ so that $\left(H_{1}, H_{2}, H_{3}\right)$ forms a harmonic vector. Consider for instance a domain $\mathcal{B}$ which intersects the plane $x=0$ in a domain which does not include the origin $y=0, z=0$. Suppose further that $\left(\xi_{1}, 0,0\right)$ and $\left(\xi_{2}, 0,0\right), \xi_{1}<0, \xi_{2}>0$ are interior points of the domain $B$. Let us now consider the function $R^{-1}, R^{2}=\left(x^{2}+y^{2}+z^{2}\right)$, which is regular in the domain $B$ since its only singularity is at $(0,0,0)$, a point outside of $\mathcal{B}$. We can choose as the remaining components $H_{2}, H_{3}$, of the vector $\left(R^{-1}, H_{2}, H_{3}\right)$ either

$$
\begin{aligned}
& H_{2}^{(1)}(X)=-\frac{y}{R(R-x)}+\operatorname{Re}[g(y+i z)] \\
& H_{3}^{(1)}(X)=-\frac{z}{R(R-x)}+\operatorname{Im}[g(y+i z)]
\end{aligned}
$$

or

$$
\begin{aligned}
& H_{2}^{(2)}(X)=\frac{y}{R(R+x)}+\operatorname{Re}[g(y+i z)], \\
& H_{3}^{(2)}(X)=\frac{z}{R(R+x)}+\operatorname{Im}[g(y+i z)]
\end{aligned}
$$

where $g$ is an arbitrary analytic function of $(y+i z)$ which is regular in the projection of $B$ on the $y, z$-plane. All these functions will be singular either at the point $\left(\xi_{1}, 0,0\right)$ or at $\left(\xi_{2}, 0,0\right)$. This situation suggests that we consider domains $\mathcal{B}$ which have the following property: Let $S_{k}$ denote $\left[\sum s(y, z)\right.$, $\left.(y, z) \in \mathcal{C}_{k}\right]$ where $\mathcal{C}_{k}$ is a curve in the $y, z$ plane and $s\left(t_{2}, t_{3}\right)$ the segment $\left[t_{1}^{(1)}<x<t_{1}^{(2)}, y=t_{2}, z=t_{3}\right]$. We assume that the domain $\mathbb{B}$ can be divided by surfaces $S_{k}$ into a finite number of subdomains $\mathbb{B}^{(v)}, \nu=1,2, \cdots, n$, 
each of which satisfies the conditions of Lemma 3.1. Thus in every domain $\mathbb{B}^{(v)}$ we may determine to a given function $H_{1}$ two harmonic functions $H_{2}^{(v)}$ and $H_{3}^{(v)}$ which are regular in $\mathcal{B}^{(v)}$ so that $H^{(v)}=\left(H_{1}, H_{2}^{(v)}, H_{3}^{(v)}\right)$ forms a harmonic vector. The boundary of each $\mathscr{B}^{(\nu)}$ consists of parts of the boundary $\mathcal{B}^{\prime}$ of $B$ and possibly of segments of one or several $S_{k}$. One can determine the $H_{2}^{(v)}, H_{3}^{(v)}$ so that they are still regular on such segments of $S_{k}$ belonging to the boundary of $\mathcal{B}^{(\nu)}$ and $\mathbb{B}^{(\mu)}$. Then:

$$
\left(H_{2}^{(\nu)}-H_{2}^{(\mu)}\right)+i\left(H_{3}^{(\nu)}-H_{3}^{(\mu)}\right)=g_{\nu}(y+i z)
$$

where $g_{\nu}(\eta)$ is a function of a complex variable which is regular in the neighborhood of $\mathcal{C}_{k}$.

Definition 1.2. A harmonic vector is said to be $B$-regular in domain $\mathbb{B}=\sum_{\nu=1}^{n} \mathcal{B}^{(\nu)}$ if it is a vector whose first component is a regular harmonic function in $\mathcal{B}$ and the components $H_{2}^{(\nu)}$ and $H_{3}^{(\nu)}$ are regular in $\mathcal{B}^{(\nu)}$, $\nu=1,2, \cdots, n$.

The analogy to the case of the theory of functions of a complex variable suggests that we introduce in the case of simply and multiply connected schlicht domains, normal vectors of the first, second, and third kind. Let $B$ be a domain whose boundary consists of finitely many sufficiently smooth surfaces $\mathcal{B}_{\nu}^{\prime}, \nu=1,2, \cdots, n$. Suppose further that $B$ satisfies the condition indicated above (see p. 490) so that to every harmonic function $H_{1}$ it is possible to determine a $B$-regular harmonic vector $\boldsymbol{H}$.

A vector $H^{(\mu)}$ whose first component $H_{1}^{(\mu)}$ assumes the value one on the boundary component $\mathcal{B}_{\mu}^{\prime}$ and vanishes on the remaining part of the boundary, is defined as a normal $B$-regular vector of the first kind.

In the two-dimensional case, using orthogonal functions, one obtains a representation of functions of the first kind. Using orthogonal functions we can obtain a representation of the harmonic vector $H^{(\mu)}$. Indeed, let $\left\{\phi_{\nu}(X)\right\}$ be a system of orthonormal functions, that is, of functions for which

$$
\left\{\phi_{\nu}, \phi_{\mu}\right\}_{\mathcal{B}} \equiv \iiint_{\mathbb{B}}\left[\frac{\partial \phi_{\nu}}{\partial x} \frac{\partial \phi_{\mu}}{\partial x}+\frac{\partial \phi_{\nu}}{\partial y} \frac{\partial \phi_{\mu}}{\partial y}+\frac{\partial \phi_{\nu}}{\partial z} \frac{\partial \phi_{\mu}}{\partial z}\right] d x d y d z=\delta_{\mu \nu}
$$

and which is complete for the class $L^{2}(B)$ of functions $H$ which are harmonic in $\mathbb{B}$ and for which $\{H, H\}_{\mathcal{B}}<\infty$. Let $k(X ; \Xi)=\sum \phi_{s}(\boldsymbol{X}) \phi_{\boldsymbol{s}}(\boldsymbol{\Xi})$ be the kernel of this system. Then

$$
\begin{aligned}
H_{1}(X) & =\sum_{s=1}^{\infty} a_{s} \phi_{s}(X)=\iint_{\mathcal{B}_{\mu}{ }^{\prime}} \frac{k(X ; \boldsymbol{Z})}{\partial n_{\Xi}} d \omega_{\Xi}, \\
a_{s} & =\iint_{\mathcal{B}_{\mu}}\left(\frac{\partial \phi_{s}}{\partial n_{\Xi}}\right) d \omega_{\Xi}
\end{aligned}
$$

where $n_{z}$ denotes the interior normal and $d \omega_{z}$ is the surface element of $\mathcal{B}_{\mu}^{\prime}$. 
Let now $\mathcal{B}^{(v)}$ be the components of $\mathcal{B}$ described above. Suppose further that there exists an $x_{0}$ such that $P_{x}\left(\mathcal{B}^{(v)}\right)$, the projection of $\mathcal{B}^{(v)}$ on the $y, z$ plane, lies in $B \cap E\left(x=x_{0}\right)$. (This last hypothesis is not essential, but simplifies the representation.) We introduce now the new coordinate system $x^{\prime}=x-x_{0}, y^{\prime}=y_{0}, z^{\prime}=z-z_{0}$, where $\left(y_{0}, z_{0}\right)$ is a point of $P_{x}\left(B^{(v)}\right)$. Further let $X^{\prime}=\left(x^{\prime}, y^{\prime}, z^{\prime}\right)$. As we shall show, the components $H_{2}^{(\mu, \nu)}, H_{3}^{(\mu, \nu)}$ can be represented in every $\mathcal{B}^{(v)}$ in the form

$$
\begin{aligned}
H_{2}^{(\mu, \nu)}= & \sum_{s=1}^{\infty} a_{s} \int_{0}^{x^{\prime}} \frac{\partial \phi_{s}}{\partial y^{\prime}} d x^{\prime} \\
H_{3}^{(\mu, \nu)}= & \sum_{s=1}^{\infty} a_{s} \int_{(0,0,0)}^{x^{\prime}}\left\{\left[\frac{\partial \phi_{s}}{\partial z^{\prime}} d x^{\prime}-\frac{\partial \phi_{s}}{\partial x^{\prime}} d z^{\prime}\right]\right. \\
& \left.+\left(\int_{0}^{x^{\prime}} \frac{\partial^{2} \phi_{s}}{\partial y^{\prime} \partial z^{\prime}} d x^{\prime}\right) d y^{\prime}-\left(\int_{0}^{x^{\prime}} \frac{\partial^{2} \phi_{s}}{\partial y^{\prime 2}} d x^{\prime}\right) d z^{\prime}\right\} .
\end{aligned}
$$

Proof. According to theorems on harmonic functions which are orthonormal in $B$, the series $\sum_{s=1}^{\infty} a_{8} \phi_{s}(X)$ converges uniformly and absolutely. From the representation

$$
\sum_{s=1}^{\infty} a_{s} \phi_{s}(X)=\iint_{\mathcal{B}^{\prime}} \frac{\partial g(X, T)}{\partial n_{T}} \sum_{s=1}^{\infty} a_{s} \phi_{s}(T) d \omega_{T}
$$

where $g$ is the Green's function of a domain $B^{*}$ which is completely contained in $B$, follows that all derivatives of $\sum_{s=1}^{\infty} a_{s} \phi_{s}(X)$ converge uniformly and absolutely in $B^{*}$. ( $\mathscr{B}^{* *}$ is the boundary of $B^{*}$.)

Since the intersection of $\mathcal{B}^{(v)}$ with the plane $x^{\prime}=0$ is the projection of $\mathbb{B}^{(v)}$ on the $y z$-plane, and the series on the right side in (3.11) converges uniformly, $H_{2}^{(\mu, \nu)}$ is defined at every point of $\mathbb{B}^{(\nu)}$, and we have $\left(\partial H_{2}^{(\mu, \nu)} / \partial x^{\prime}\right)$ $=\left(\partial H_{1}^{(\mu, \nu)} / \partial y^{\prime}\right)$. For the derivatives of $H_{3}^{(\mu, \nu)}$ we obtain

$$
\begin{aligned}
& \frac{\partial H_{3}^{(\mu, \nu)}}{\partial x^{\prime}}=\frac{\partial H_{1}^{(\mu, \nu)}}{\partial z^{\prime}}=\sum_{s=1}^{\infty} a_{s} \frac{\partial \phi_{s}}{\partial z^{\prime}}, \\
& \frac{\partial H_{3}^{(\mu, \nu)}}{\partial y^{\prime}}=\frac{\partial H_{2}^{(\mu, \nu)}}{\partial z^{\prime}}=\sum_{s=1}^{\infty} a_{s} \int_{0}^{x^{\prime}} \frac{\partial^{2} \phi_{s}}{\partial y^{\prime} \partial z^{\prime}} d x^{\prime}, \\
& \frac{\partial H_{3}^{(\mu, \nu)}}{\partial z^{\prime}}=-\sum_{s=1}^{\infty}\left(a_{s} \frac{\partial \phi_{s}}{\partial x^{\prime}}+a_{s} \int_{0}^{x^{\prime}} \frac{\partial^{2} \phi_{s}}{\partial y^{\prime 2}} d x^{\prime}\right) \text {. } \\
& \left\{\phi_{s}, \int_{0}^{x^{\prime}} \frac{\partial \phi_{s}}{\partial y^{\prime}} d x^{\prime}, \int_{(0,0,0)}^{x^{\prime}}\left\{\frac{\partial \phi_{s}}{\partial z^{\prime}} d x^{\prime}+\left(\int_{0}^{x^{\prime}} \frac{\partial^{2} \phi_{s}}{\partial y^{\prime} \partial z^{\prime}} d x^{\prime}\right) d y^{\prime}\right.\right. \\
& \left.\left.-\left(\frac{\partial \phi_{s}}{\partial x^{\prime}}+\int_{0}^{x^{\prime}} \frac{\partial^{2} \phi_{s}}{\partial y^{\prime 2}} d x^{\prime}\right) d z^{\prime}\right\}\right\}
\end{aligned}
$$


is a harmonic vector;

$$
\begin{aligned}
\phi_{s} d x+\left\{\int_{0}^{x^{\prime}} \frac{\partial \phi_{s}}{\partial y^{\prime}} d x^{\prime}\right\} d y+\left\{\int_{(0,0,0)}^{x^{\prime}} \frac{\partial \phi_{s}}{\partial z^{\prime}} d x^{\prime}+\right. & \left(\int_{0}^{x^{\prime}} \frac{\partial^{2} \phi_{s}}{\partial y^{\prime} \partial z^{\prime}} d x^{\prime}\right) d y^{\prime} \\
& \left.-\left(\frac{\partial \phi_{s}}{\partial x^{\prime}}+\int_{0}^{x^{\prime}} \frac{\partial^{2} \phi_{s}}{\partial y^{\prime 2}} d x^{\prime}\right) d z^{\prime}\right\} d z
\end{aligned}
$$

is therefore a complete differential. Since the series (3.12) converges uniformly the integrand in the right-hand side of (3.12) is a complete differential, the series converges uniformly and absolutely, and $H_{1}, H_{2}^{(\mu, \nu)}, H_{3}^{(\mu, \nu)}$ is a harmonic vector. A harmonic vector whose $x$-component becomes infinite as $\left[(x-\xi)^{2}\right.$ $\left.+(y-\eta)^{2}+(z-\zeta)^{2}\right]^{1 / 2}$ at a point $(\xi, \eta, \zeta) \in \mathcal{B}^{(v)}$ will be defined as a normal $B$-regular vector of the third kind. Using orthogonal functions one can derive a representation similar to (3.6), (3.7), (3.8), for these vectors. In an analogous manner we can define normal $B$-regular vectors of the second kind and derive a representation by the use of orthonormal functions.

3. Integrals of algebraic functions. The analogy to the case of one complex variable suggests that we form integrals of harmonic vectors whose components are algebraic functions.

In the following,

$$
\begin{aligned}
& H=\left(H_{1}, H_{2}, H_{3}\right)=\mathbf{R}_{3}\left(f, \mathcal{L}, X_{0}, 0\right), \quad f=p(u, \zeta) / q(u, \zeta), \\
& H_{1}=\int_{\mathcal{L}} f(u, \zeta) \frac{d \zeta}{\zeta}, \quad H_{2}=\frac{i}{2} \int_{\mathcal{L}}\left(\zeta+\zeta^{-1}\right) f(u, \zeta) d \zeta / \zeta, \\
& H_{3}=\frac{1}{2} \int_{\mathcal{L}}\left(\zeta-\zeta^{-1}\right) f(u, \zeta) d \zeta / \zeta, \quad \boldsymbol{X} \in V\left(\boldsymbol{X}_{0}\right) \text {, }
\end{aligned}
$$

will denote a harmonic vector with a rational $B_{3}$-associate $f$. Here $\mathcal{L}$ is a conveniently chosen closed curve in the $\zeta$-plane, and $U\left(\boldsymbol{X}_{0}\right)$ some neighborhood of $X_{0}$. Forming "integrals" we can then consider two classes of functions.

I. Let $J=\mathrm{E}[x=x(\sigma), y=y(\sigma), z=z(\sigma)]$ be a segment of a curve which begins at $X_{1}=X\left(s_{1}\right)$ and ends at $X=X(s), s_{1}<s$. It is assumed that $J$ lies in the regularity domain of $\boldsymbol{H}$. Then

$$
\begin{aligned}
\Lambda\left(X, X_{1}\right) & =\int_{J} H(X) \cdot d X \\
& =\int_{\sigma=s_{1}}^{s}\left[H_{1} \frac{\partial x}{\partial \sigma}+H_{2} \frac{\partial y}{\partial \sigma}+H_{3} \frac{\partial z}{\partial \sigma}\right] d \sigma
\end{aligned}
$$

is a harmonic function of $X$ as well as of $X_{1}$. The integrand is complete differential and therefore $\Lambda\left(X, X_{1}\right)$ does not change its value, if $X_{1}$ and $X$ are fixed and $J$ varies continuously in the regularity domain of $H$.

II. Let $B^{\prime}$ be a sufficiently small "cylindrical surface" 


$$
\mathbb{B}^{\prime}=\mathrm{E}\left[x=x(s, v), y=y(s, v), z=z(s, v), 0 \leqq v \leqq 1, s_{0} \leqq s \leqq s_{1}\right]
$$

where $x(s, 0)=x(s, 1), y(s, 0)=y(s, 1), z(s, 0)=z(s, 1)$ are bounded by the curves $\sigma_{K}=\mathrm{E}\left[x=x\left(s_{K}, v\right), y=y\left(s_{K}, v\right), z=z\left(s_{K}, v\right), 0 \leqq v \leqq 1\right], K=1,2$.

Then

$$
V\left(\sigma_{1}, \sigma_{2}\right)=\iint_{\mathcal{B}^{\prime}}\left[H_{1} \frac{\partial(y, z)}{\partial(s, v)}+H_{2} \frac{\partial(z, x)}{\partial(s, v)}+H_{3} \frac{\partial(x, y)}{\partial(s, v)}\right] d s d v
$$

are functions of curves $\sigma_{1}, \sigma_{2}$. We note that functions of curves, $V$, generated in the manner indicated above by harmonic vectors have been introduced for the first time by Volterra, see [13], [14].

REMARK 3.1. The case where one of the curves, say $\sigma_{2}$, degenerates to a point and $\mathcal{B}^{\prime}$ becomes a domain bounded by one curve, $\sigma_{1}$, can be considered as a special case of functions $V$.

In the present paper we shall study only the functions $\Lambda\left(X, X_{1}\right)$ indicated in $I$. Certain of our considerations can be generalized to functions $V$.

Since it has been shown in $\S \S$ I.2, I.3 that the $H_{k}$ are defined in the large, the $\Lambda\left(X, X_{1}\right)$ 's are also defined in the large. Let $R^{(0)}$ denote the $R$-manifold of the $H_{k}$. The R-manifold $R$ of $\Lambda\left(X, X_{1}\right)$ can be obtained by cutting in a convenient manner $R^{(0)}$ and by attaching new space sheets $\left({ }^{28}\right) R^{(1)}, R^{(2)}, \cdots$. In general, $R=\sum_{\nu=-\infty}^{\infty} R^{(\nu)}$ consists of infinitely many sheets. The study of its structure is connected with the study of "periods" of (3.17); these questions will be discussed in the next section.

It should perhaps be added that

$$
\Lambda\left(X, X_{1}\right)=\int_{X_{1}}^{X} H \cdot d X=\frac{1}{2 \pi} \int_{\mathcal{L}} \int_{u_{1}}^{u} f(u, \zeta) d u \frac{d \zeta}{\zeta}
$$

has a $\mathrm{B}_{3}$-associate which is an algebro-logarithmic function. As it can be shown, it has the property that considered as a function of $x, y$, and $z$, respectively, it satisfies an ordinary differential equation. See [2].

REMARK 3.2. In this chapter we established that there exist certain Rmanifolds on which single-valued harmonic functions have been defined. The further question which arises is to establish the existence of further functions which are defined and single-valued either on the closed R-manifold, or in a subdomain $\mathcal{B}$ (including some branch-lines) of the R-manifold, as well as to study the properties of these functions. These problems can be attacked by generalizing the classical procedures (see Weyl [16]), in particular considering functions sufficiently of ten differentiable, for which

$$
\iiint_{\mathscr{B}}\left[\left(\frac{\partial u}{\partial x}\right)^{2}+\left(\frac{\partial u}{\partial y}\right)^{2}+\left(\frac{\partial u}{\partial z}\right)^{2}\right] d x d y d z
$$

${ }^{(28)}$ Each $R^{(v)}$ is an identical copy of $R^{(0)}$. We identify it by a different $\nu$, since it becomes (after being conveniently cut) a space sheet of new covering R-manifold. 
becomes minimum, and which assumes prescribed values on the boundary of $B$ or has necessary discontinuities.

4. Relations between the function $\Lambda\left(X, X_{1}\right)$ and integrals of algebraic functions of a complex variable. Abel's theorem in the theory of integrals of algebraic functions of one complex variable establishes a relation between two different classes of functions: it shows that a sum of integrals of algebraic functions with conveniently chosen arguments is equal to an algebraic function. Here, the values of the arguments are connected by an algebraic relation. In the preceeding section, we introduced a class of harmonic functions $\Lambda^{(\nu)}\left(X, X_{1}\right)$ which one obtains by forming $\int_{X_{1}}^{X} H^{(\nu)} \cdot d X$, where $H^{(\nu)}$ is an algebraic harmonic vector. In this section we shall show that for these functions a theorem (which to a certain extent can be considered as an analogue to Abel's theorem) will hold. Namely we shall show that a sum $\sum_{X^{(\kappa)}}^{X^{(\kappa+1)}} H^{(\nu)} \cdot d \boldsymbol{X}$ is equal to a certain sum of integrals of algebraic functions of one complex variable; here the limits of integration appearing in both sums are connected by an algebraic relation.

The main idea underlying the derivation of the above relation is to evaluate an iterated integral of a conveniently chosen integrand and to carry out the integration in two different orders. In one case it then yields a sum $\sum \Lambda^{(\nu)}\left(\boldsymbol{X}^{(s)}, \boldsymbol{X}^{(k)}\right)$ (see 3.17), in the other $\sum_{\mu} \int_{\zeta^{(k)}}^{\zeta^{(\kappa+1)}} a^{(\mu)}(\zeta) d \zeta$, where $a^{(\mu)}$ are algebraic functions of a complex variable.

In order to formulate our results in a more precise manner we introduce certain notions.

Let $\mathcal{L}$ be as in $\S I I .2$, a closed simple oriented curve in the $\zeta$-plane which includes neither the origin nor double points in its interior and let $J=\mathrm{E}[x=x(\mathrm{~s})$, $y=y(\mathrm{~s}), z=z(\mathrm{~s}), 0 \leqq \mathrm{~s} \leqq 1], x(0)=x(1), y(0)=y(1), z(0)=z(1)$, be a closed simple sufficiently smooth oriented curve in the schlicht $x, y, z$-space.

Let

$$
\frac{p(u, \zeta)}{q(u, \zeta)}=\frac{\zeta^{N-M} P(\zeta ; X)}{Q(\zeta ; X)}
$$

be a rational function of $u$ and $\zeta$ as introduced in $\S$ II.2 (see (2.4), (2.12)). For every fixed value of $\zeta$ the relation

$$
q\left[\left(x+2^{-1} i y\left(\zeta+\zeta^{-1}\right)+2^{-1} z\left(\zeta-\zeta^{-1}\right)\right), \zeta\right]=0
$$

which can be written also in the form

$$
x+2^{-1} i y\left(\zeta+\zeta^{-1}\right)+2^{-1} z\left(\zeta-\zeta^{-1}\right)=u^{(\nu)}(\zeta), \quad \nu=1,2, \cdots, n,
$$

defines $n$ straight lines $N^{(v)}(\zeta)$, in the $x, y, z$-space. If $\zeta$ ranges over the curve $\mathcal{L}$,

$$
\sum_{\nu=1}^{n} \sum_{\zeta \in \mathcal{L}} N^{(\nu)}(\zeta)
$$


forms a ruled surface in the $x, y, z$-space.

Let us assume that $J$ lies completely in the regularity domain of $H_{k}(X)$ $=R_{k}\left(\zeta^{(v)} ; X\right), k=1,2,3$ (see (2.13a) and $(2.13 \mathrm{~b})$ ), in particular that $J$ has no points in common with the branch line $\mathcal{S}$ of $H_{k}(\boldsymbol{X})$.

We denote by $X^{(\kappa)}, \kappa=1,2, \cdots, s$, the intersection points of $J$ with the surfaces (3.24) and assume that $s<\infty$. The points $X^{(\kappa)}$ decompose $J$ into $s$ segments, $J_{k}$, whose end points are $X^{(\kappa)}$ and $X^{(\kappa+1)}, X^{(\bullet+1)}=X^{(1)}$.

Lемма 3.3. To every segment $J_{k}$ there corresponds a sequence, say $T_{k}(\mathcal{L})$, of the roots $\zeta^{(v)}(X)$ of $Q(\zeta ; X)=0$ (see (2.14)) such that the roots

$$
\zeta^{\left(\nu_{\mu}\right)}(X), \quad \nu_{\mu} \in T_{\kappa}(\mathcal{L})
$$

lie inside of $\mathcal{L}$ and the remaining roots lie outside of $\mathcal{L}$.

Proof. It is at first clear that if $X$ is an interior point of $J_{k}$ no roots $\zeta^{(v)}(X)$ can lie on $\mathcal{L}$. If a point, say $\zeta^{(v)}(X)$, should lie on $\mathcal{L}$, then the relation

$$
\begin{aligned}
q\left[\left(x+2^{-1} i y\left(\zeta^{(\nu)}(X)\right.\right.\right. & \left.+\left(\zeta^{(v)}(X)\right)^{-1}\right) \\
& \left.\left.+2^{-1} z\left(\zeta^{(\nu)}(X)-\left(\zeta^{(\nu)}(X)\right)^{-1}\right)\right), \zeta^{(\nu)}(X)\right]=0
\end{aligned}
$$

would hold and therefore $\Im$ would intersect the surface (3.24) at this point $X$, which is impossible: the intersection points of $J$ and (3.24) are by definition end points of the intervals $J_{k}$.

Further, since $\zeta^{(\nu)}(X)$ vary continuously as $X$ moves along $J$, a root $\zeta^{(v)}(X)$ can move in or out of $\mathcal{L}$ only for a value of $X$ for which $\zeta^{(\nu)}(X)$ lies on $\mathcal{L}$. By definition, these points are end points $X^{(\kappa)}$ of the intervals $J_{k}$ and therefore we can associate the $T_{k}(\mathcal{L})$ described in the lemma with every interval $J_{k}$.

For every value of $\zeta\left({ }^{29}\right)$,

$$
\begin{aligned}
J^{\prime}(\zeta)=\mathrm{E}\left[u=x(\mathrm{~s})+2^{-1} i y(\mathrm{~s})\left(\zeta+\zeta^{-1}\right)\right. & \\
& \left.+2^{-1} z(\mathrm{~s})\left(\zeta-\zeta^{-1}\right), 0 \leqq \mathrm{~s} \leqq 1\right\rfloor
\end{aligned}
$$

represents a closed oriented curve in the $u$-plane. Let us denote by $\zeta^{(\mu)}$, $\mu=1,2, \cdots, s^{\prime}$ points of $\mathcal{L}$ which correspond to $X^{(\kappa)}$ (in 3.22)). Since $\mathcal{L}$ is an oriented curve the $\zeta^{(\mu)}$ can be numerated in the order which we meet them moving continuously along $\mathcal{L}$. $\mathcal{L}$. will then be decomposed into $s^{\prime}$ parts $\mathcal{L}_{\mu}, \mathcal{L}_{\mu}$ having the end points $\zeta^{(\mu)}$ and $\zeta^{(\mu+1)}, \zeta^{(1)}=\zeta^{\left(0^{\prime}+1\right)}$.

REMARK 3.3. If we denote by $D(\mathcal{L})$ the set of those points $\boldsymbol{X}$ for which the equation (3.22) possesses two roots $\zeta$ which lie on the curve $\mathcal{L}$, then

$$
s^{\prime}=s
$$

if the curve $J$ does not include any points of $D(\mathcal{L})$. Otherwise $s^{\prime}<s$.

If we now denote by $u^{(v)}(\zeta), \nu=1,2, \cdots, n$, the roots of the equation

$\left.{ }^{(29}\right)$ We note that for some values of $\zeta$ this curve may degenerate into an open segment which is described twice when $s$ varies from $s=0$ to $s=1$. 


$$
q(u, \zeta)=0
$$

then again we can associate with every segment $\mathcal{L}_{\mu}$ a set, say $\tau_{\mu}(\mathcal{J})$, so that the $u^{(\kappa)}(\zeta), \kappa \in \tau_{\mu}(\Im)$, lie inside the domain bounded by $\Im^{\prime}(\zeta), \zeta \in \mathcal{L}_{\mu}$, while the remaining $u^{(\kappa)}(\zeta)$ lie outside $J^{\prime}(\zeta)$.

Theorem 3.1. Let $\boldsymbol{H}=\mathbf{R}_{3}\left(p / q, \mathcal{L}, \boldsymbol{X}_{0}, 0\right), \boldsymbol{X}_{0} \neq 0$ (see (3.2)) be a harmonic vector with a rational $\mathrm{B}_{3}$-associate $p / q$ which vector is defined on the $\mathrm{R}$-manifold $R$ with $2 n$ sheets. See (2.58). Its components are $2 N$-valued functions $\left({ }^{30}\right)$ whose branches we shall denote by $H_{\boldsymbol{t}}^{(\nu)}(X), k=1,2,3, \nu=1,2, \cdots, 2 N$ (see (2.13a), (2.13b)).

If $J$ is a closed simple oriented curve in the schlicht $x, y, z$-space as described above, then

$$
\begin{aligned}
\sum_{\kappa=1}^{s} \sum_{\nu \in T_{k}(\mathcal{L})} \Lambda^{(\nu)}\left(X^{(\kappa+1)}, X^{(\kappa)}\right) & \\
= & \sum_{k=1}^{s^{\prime}} \sum_{\mu \in T_{k}(J)} \int_{\zeta^{(k)}}^{\zeta(\alpha+1)}\left[\frac{p(u, \zeta)}{\zeta \partial q(u, \zeta) / \partial u)}\right]_{u=u^{(\mu)}(\zeta)} d \zeta,
\end{aligned}
$$

where $\Lambda^{(v)}\left(X, X_{1}\right)=\int_{X_{1}}^{X} H^{(v)} \cdot d X$, see (2.59), $u^{(\mu)}(\zeta)$ are the roots of (3.28) and $T_{\kappa}(\mathcal{L})$ and $\tau_{\kappa}(\mathcal{J})$ are the sequences described above. $X^{(\kappa)}$ and $\zeta^{(\mu)}$ are connected by the equation (3.22).

Proof. As we indicated before, (3.29) is obtained by evaluating the sum of three integrals,

$$
\begin{aligned}
S=\int_{\mathcal{J}}\left(\int_{\mathcal{L}} \frac{p(u, \zeta)}{q(u, \zeta)} \frac{d \zeta}{\zeta}\right) d x+i \int_{\mathcal{J}} & \left(\int_{\mathcal{L}} \frac{p(u, \zeta)}{q(u, \zeta)} \frac{1+\zeta^{-2}}{2} d \zeta\right) d y \\
& +\int_{\zeta}\left(\int_{\mathcal{L}} \frac{p(u, \zeta)}{q(u, \zeta)} \frac{1-\zeta^{2}}{2} d \zeta\right) d z
\end{aligned}
$$

and interchanging the order of integration. Since $J=\sum_{k=1}^{s} J_{k}$ we can replace in (3.30) $\int \mathfrak{J}$ by $\sum_{k=1} \int J_{\mathfrak{K}}$. According to the results of $\S I I .2$, since $\mathcal{L}$ does not include the origin and has no double points, we have for every $X \in \mathfrak{J}_{k}$

$$
\int_{\mathcal{L}} \frac{p(u, \zeta)}{q(u, \zeta)} \frac{d \zeta}{\zeta}=\sum_{\nu \in T_{\boldsymbol{K}}\left(\mathfrak{J}^{\prime}\right)} H_{1}^{(\nu)}(X), \quad H_{1}^{(\nu)}(X)=R_{1}\left(\zeta^{(\nu)}(X), X\right),
$$

as given by (2.13a) and (2.13b). Analogously by (3.2)

$$
\begin{aligned}
& i \int_{\mathcal{L}} \frac{p(u, \zeta)}{q(u, \zeta)} \frac{1+\zeta^{-2}}{2} d \zeta=\sum_{\nu \in T_{K}\left(J_{)}\right.} H_{2}^{(\nu)}(X) \\
& \int_{\mathcal{L}} \frac{p(u, \zeta)}{q(u, \zeta)} \frac{1-\zeta^{-2}}{2} d \zeta=\sum_{\nu \in T_{\mathbf{K}}\left(J_{)}\right.} H_{3}^{(\nu)}(X) .
\end{aligned}
$$

(30) In the schlicht $x, y, z$-space. 
Thus we obtain

$$
\begin{array}{rlrl}
S & =\sum_{\kappa=1}^{s} \int_{J_{\kappa}} \sum_{\nu \in T_{\kappa}(\mathcal{L})}\left[H_{1}^{(\nu)} d x+H_{2}^{(\nu)} d y+H_{3}^{(\nu)} d z\right] \\
& =\sum_{\kappa=1}^{s} \sum_{\nu \in T_{\kappa}(\mathcal{L})} \Lambda^{(\nu)}\left(X^{(\kappa+1)}, X^{(\kappa)}\right) ; & X^{(\bullet+1)}=X^{(1)}
\end{array}
$$

As we shall show subsequently, the double integral taken over the absolute value of the integrand exists; therefore we can interchange the order of integration to obtain

$$
\begin{aligned}
S & =\sum_{\mu=1}^{s^{\prime}} \int_{\mathcal{L}_{\mu}}\left[\int_{J^{\prime}(\zeta)} \frac{p(u, \zeta)}{q(u, \zeta)} d u\right] \frac{d \zeta}{\zeta} \\
& =\sum_{\mu=1}^{s^{\prime}} \int_{\mathcal{L}_{\mu}} \sum_{\nu \in \epsilon_{\tau \mu}(\zeta)}\left[\frac{p(u, \zeta)}{\zeta \partial q(u, \zeta) / \partial u}\right]_{u=u(\nu)(\zeta)} d \zeta \\
& =\sum_{\mu=1}^{s^{\prime}} \sum_{\nu \in \tau_{\mu}\left(J_{)}\right.} \int_{\zeta^{(\mu)}}^{\zeta^{(\mu+1)}}\left[\frac{p(u, \zeta)}{\zeta \partial q(u, \zeta) / \partial u}\right]_{u=u^{(\nu)}(\zeta)} d \zeta,
\end{aligned}
$$

which yields the right-hand side of (3.29).

It still remains to show that the integrands in (3.30) are absolutely integrable. Let us consider one of these integrals, say the first one. According to our hypotheses (see p. 495) the curve $\mathcal{L}$ does not include the origin; $p(u, \zeta)$ is everywhere bounded for $X \in J$; it is therefore sufficient to show that

$$
\int_{J} \int_{\mathcal{L}}\left|\frac{d x d \zeta}{q(u, \zeta)}\right|
$$

exists. $q(u, \zeta)$ vanishes only at the intersection points $X^{(\kappa)}$ of $J$ with the surface (3.24). There are only finitely many points $X^{(\kappa)}$ (see p. 496). We shall consider the behavior of the integrand in the neighborhood of one of the $\boldsymbol{X}^{(\kappa)}$, say at $\boldsymbol{X}=\boldsymbol{X}^{(1)}=\left(x^{(1)}, y^{(1)}, z^{(1)}\right)$ which lies on one of the $\mathcal{N}^{(v)}(\zeta)$, say on $\mathcal{N}^{(1)}\left(\zeta_{1}\right)$. See (3.23).

Let the curve $\mathcal{L}$ in the neighborhood of $\zeta_{1}$ be given by

$$
\zeta=\zeta(\tau), \quad-\tau_{2} \leqq \tau \leqq \tau_{2}, \quad \zeta_{1}=\zeta(0)
$$

and the curve $\boldsymbol{J}$ in the neighborhood of $\boldsymbol{X}^{(1)}$ by $\boldsymbol{X}=\boldsymbol{X}(s)$, that is,

$$
x=x(s), \quad y=y(s), \quad z=z(s), \quad-s_{2} \leqq s \leqq s_{2}, \quad X^{(1)}=X(0) .
$$

We can choose the parametrizations so that $\left|\zeta^{\prime}(\tau)\right|,\left|x^{\prime}(s)\right|,\left|y^{\prime}(s)\right|,\left|z^{\prime}(s)\right|$ are uniformly bounded in the above neighborhoods.

Since $X^{(1)}$ lies outside the branchlines of the R-manifold (2.14), according to p. 654 of [2], all roots $\zeta^{(\nu)}\left(X^{(1)}\right)$ are different, and since thus in 


$$
q\left[x+\frac{i y}{2}\left(\zeta+\zeta^{-1}\right)+\frac{1}{2} z\left(\zeta-\zeta^{-1}\right), \zeta\right]=\prod_{\nu=1}^{N}\left(\zeta-\zeta^{(\nu)}(X)\right)
$$

only one factor vanishes for $\boldsymbol{X}=\boldsymbol{X}^{(1)}$, it suffices to consider the integral

$$
P=\int_{s=0}^{s_{2}} \int_{\tau=0}^{\tau_{2}}\left|\frac{d s d \zeta(\tau)}{\zeta(\tau)-\zeta^{(\nu)}(X(s))}\right| .
$$

Let $\zeta=\xi+i \eta, \zeta^{(v)}(\boldsymbol{X}(s))=\xi^{(\nu)}(\boldsymbol{X}(s))+i \eta^{(\nu)}(\boldsymbol{X}(s))$. We assume that the parametrization is done in such a way that $C|d \xi(\tau)| \geqq|d \zeta(\tau)|, C$ being a suitable constant.

$$
P \leqq 4 C \int_{s=0}^{s_{2}} \int_{\xi=0}^{\xi_{2}} \frac{d s d \xi}{\left|\xi-\xi^{(v)}(X(s))\right|} .
$$

If we assume further that $s_{2}$ is so small that for $-s_{2} \leqq s \leqq s_{2}$

$$
\left|\xi_{2}\right|>2\left|\zeta^{(\nu)}(X(s))\right|
$$

then

$$
P \leqq 4 C \int_{0}^{s_{2}} d s\left|\lg \xi^{(v)}(X(s))\right|+O(1)
$$

Now let us consider

$$
J=\int_{0}^{s_{2}}\left|\lg \xi^{(\nu)}(X(s))\right| d s .
$$

$\xi^{(\nu)}(X)$ is an analytic function of $x, y, z$, while according to our hypotheses $x(s)$ can be represented in the neighborhood of $s=0$ as $x(s)=\alpha_{1} s+\alpha_{2}(s) s^{2}$, and $y(s)$ and $z(s)$ similarly. Therefore $\xi^{(\nu)}(X(s))=A s^{n}+B(s) s^{n+1}, A \neq 0$, where $B(s)$ is uniformly bounded in the neighborhood of $s=0$, from which it follows that the integral $J$ exists.

\section{SOME RELATIONS BETWEEN FUNCTIONS $H(\boldsymbol{X})$ AND $\Phi(\boldsymbol{x})$ BELONGING TO DIFFERENT CLASSES}

1. The operator $P_{3}$ transforming harmonic functions into solutions of more general linear differential equations. The next step in the development of our method consists in the study of operators $\mathbf{P}_{3}$ which transform harmonic functions in three variables into solutions of equations

$$
\Delta u+F(x, y, z) u=0
$$

(see problem II, §I.1). As indicated on p. 464, we shall study this problem for equations of the form (1.3), that is, where $F=C\left(r^{2}\right)$ is an entire function of $r^{2}=x^{2}+y^{2}+z^{2}$. 
In a previous paper [9] it has been shown that for every equation (1.3) there exists a generating function

$$
\Omega(r, \tau)=1+\sum_{n=1}^{\infty} \tau^{2 n} b^{(n)}\left(r^{2}\right)
$$

such that, in analogy to (1.3), the operator

$$
\mathrm{P}_{8}[H(X)]=\int_{-1}^{1} \Omega(r, \tau) H\left[X\left(1-\tau^{2}\right)\right] d \tau
$$

generates solutions of equation (1.5) from harmonic functions $H(\boldsymbol{X})$ of three variables. For our purposes it is useful to modify this operator slightly.

LEMMA 4.1. The operator

$$
\begin{gathered}
\mathrm{p}_{3}[G(X)]=G(X)+\sum_{n=1}^{\infty} B^{(n)}\left(r^{2}\right) \int_{0}^{1}\left(1-\sigma^{2}\right)^{n-1} \sigma^{2} G\left(\sigma^{2} X\right) d \sigma \\
B^{(n)}\left(r^{2}\right)=\frac{\Gamma(n+1 / 2)}{\Gamma(n) \Gamma(1 / 2)} b^{(n)}\left(r^{2}\right)
\end{gathered}
$$

generates solutions $\Phi(X)=p_{3}[G(X)]$ of (1.6) from harmonic functions $G(X)$. (Here $G$ and $H$ are connected by (4.8).)

Proof. Let

$$
H(X)=\sum_{p=0}^{\infty} \sum_{M+N+P=p} a_{M N P} x^{M} y^{N} z^{P} .
$$

Then (4.3) can be written in the form

(4.6) $\quad \mathrm{P}_{3}[H(X)]=\sum_{p=0}^{\infty} \sum_{M+N+P=p} \dot{a}_{M N P} x^{M} y^{N} z^{P} \int_{\tau=-1}^{1} \Omega(r, \tau)\left(1-\tau^{2}\right)^{p} d \tau$.

On the other hand, by (4.2)

$$
\begin{gathered}
\begin{aligned}
\int_{\tau=-1}^{1} \Omega(r, \tau)\left(1-\tau^{2}\right)^{p} d \tau & =\sum_{n=0}^{\infty} b^{(n)}\left(r^{2}\right) \int_{\tau=-1}^{1} \tau^{2 n}\left(1-\tau^{2}\right)^{p} d \tau \\
& =\sum_{n=0}^{\infty} \frac{\Gamma(n+1 / 2) \Gamma(p+1)}{\Gamma(n+p+3 / 2)} b^{(n)}\left(r^{2}\right) \\
& =\frac{\Gamma(p+1) \Gamma(1 / 2)}{\Gamma(p+3 / 2)}\{1 \\
+\sum_{n=1}^{\infty} b^{(n)}\left(r^{2}\right) \frac{\Gamma(n+1 / 2)}{\Gamma(1 / 2)} & \left.\int_{s_{1}=0}^{1} \cdots \int_{s_{n-1}=0}^{s_{n-2}} \int_{s_{n}=0}^{s_{n-1}} s_{n}^{p+1 / 2} d s_{n} \cdots d s_{1}\right\}
\end{aligned}
\end{gathered}
$$


Thus by writing $\left({ }^{31}\right)$

$$
G(X) \equiv G(x, y, z)=\sum_{M, N, P}^{\infty} a_{M N P} \frac{\Gamma(p+1) \Gamma(1 / 2)}{\Gamma(p+3 / 2)} x^{M} y^{N} z^{P}(p=M+N+P)
$$

$$
=\int_{T=0}^{1} T^{-1 / 2} H[X(1-T)] d T
$$

we obtain

$$
\begin{gathered}
\mathrm{p}_{3}[G]=\left\{G(X)+\sum_{n=1}^{\infty} \frac{\Gamma(n+1 / 2)}{\Gamma(1 / 2)} b^{(n)}\left(r^{2}\right) \int_{s_{1}=0}^{1} \ldots\right. \\
\left.\cdot \int_{s_{n-1}=0}^{s_{n-2}} \int_{s_{n}=0}^{s_{n-1}} s_{n}^{1 / 2} G\left(s_{n} X\right) d s_{n} d s_{n-1} \cdots d s_{1}\right\} .
\end{gathered}
$$

By noting that

$$
\begin{aligned}
\int_{s_{1}=0}^{1} \cdots \int_{s_{n-1}=0}^{s_{n-2}} & \int_{s_{n}=0}^{s_{n-1}} s_{n}^{1 / 2} G\left(s_{n} X\right) d s_{n} d s_{n-1} \cdots d s_{1} \\
& =\frac{1}{\Gamma(n)} \int_{s=0}^{1}(1-s)^{n-1} s^{1 / 2} G(s X) d s \\
& =\frac{2}{\Gamma(n)} \int_{\sigma=0}^{1}\left(1-\sigma^{2}\right)^{n-1} \sigma^{2} g\left(\sigma^{2} X\right) d \sigma
\end{aligned}
$$

we obtain (4.4) with the $B^{(n)}$ defined by (4.4a).

Since in a sufficiently small neighborhood of the origin the series (4.2) and (4.5) converge uniformly and absolutely, the interchanging of the orders of summations, as well as that of summation and integrations are admissible. It should be noted that in the case of differential equations (4.1) where $F(x, y, z)$ is an entire function of three complex variables, we obtain from harmonic function $H(x, y, z)$, solutions of (4.1) by forming the expression

$$
H(x, y, z)-\iiint_{B} \frac{H(\xi, \eta, \zeta) F(\xi, \eta, \zeta) d \xi d \eta d \zeta}{\left[(x-\xi)^{2}+(y-\eta)^{2}+(z-\zeta)^{2}\right]^{1 / 2}}+\cdots
$$

where the integration has to be taken by a conveniently chosen domain $\mathbb{B}$.

We wish to show that every solution of $T(\Phi)=0$, see (1.5), can be represented in a sufficiently small neighborhood of the origin in the form $\Phi=p_{3}(H)$.

For every equation $T(\Phi)=0$, one can determine a sufficiently small $r_{0}$ such that for every sphere $S=E\left[x^{2}+y^{2}+z^{2}<\rho^{2}\right], 0<\rho \leqq r_{0}$, there exists a function $A(r, \theta, \phi)$ possessing the following property: for every solution

(i) $G(X)$ is a harmonic function. 
$\Phi(\boldsymbol{X})$ which is regular in $\mathcal{S}^{\prime}+\mathcal{S}, \mathcal{S}^{\prime}=\mathrm{E}\left[x^{2}+y^{2}+z^{2}=\rho^{2}\right],|\Phi(r, \theta, \phi)|$ $\leqq A(r, \theta, \phi) \max _{0 \leqq \phi \leqq 2 \pi, 0 \leqq \theta \leqq \pi}|\Phi(\rho, \theta, \phi)|, r<\rho$. Therefore, according to Theorem 4.1, p. 429 of [9] every real solution $\Phi$ of $T(\Phi)=0$ can be represented in a sufficiently small sphere in the form

$$
\Phi(X)=p_{3}(H)=H(X)+\sum_{n=1}^{\infty} B^{(n)}\left(r^{2}\right) \int_{\sigma=0}^{1}\left(1-\sigma^{2}\right)^{n-1} \sigma^{2} H\left(\sigma^{2} X\right) d \sigma .
$$

Definition 4.1. The function $H(X)$ which when substituted in (4.12) generates a given solution $\Phi$ of $T(\Phi)=0$ is defined as the $p_{3}$-associate of $\Phi$.

According to our previous discussion and the considerations of $\$ 4$, p. 428 of [9], for every solution $\Phi$ which is regular at the origin there exists one and only one $\mathbf{p}_{3}$-associate which is regular at the origin.

From the representation (4.12) follows:

LEMmA 4.2. If $H$ is the $\mathrm{p}_{3}$-associate of $\Phi(X)$, that is, if $\Phi=\mathrm{p}_{3}(H)$, then in the characteristic space $\mathcal{C}_{3}$ the relation

$$
\Phi\left[i\left(y^{2}+z^{2}\right)^{1 / 2}, y, z\right]=H\left[i\left(y^{2}+z^{2}\right)^{1 / 2}, y, z\right]
$$

holds.

Proof. (4.13) follows immediately from the fact that $B^{(n)}(0)=0$ (see (4.20) p. 425 of [9]).

Definition 4.2. As before we define

$$
\chi\left(Z, Z^{*}\right)=\Phi\left[2\left(Z Z^{*}\right)^{1 / 2},-i\left(Z+Z^{*}\right),\left(Z-Z^{*}\right)\right]
$$

as the $\mathbf{C}_{3}$-associate of $\Phi$.

REMARK 4.1. Using the symbols $\mathbf{C}_{3}$ and $\mathrm{p}_{3}$, Lemma 4.2 can be written in the form

$$
\mathrm{C}_{3}^{-1}(\Phi)=\mathbf{C}_{3}^{-1}\left[\mathrm{~b}_{3}^{-1}(\Phi)\right] .
$$

This, as well as the fact that the operator $\mathbf{p}_{3}$ preserves many properties of functions to which it is applied, justifies the classification of functions $\Phi$ according to the behavior of their $\mathrm{p}_{3}$-associates.

2. Solutions $\Phi$ of $T(\Phi)=0$ generated by rational and algebraic $p_{3}$-associates.

LEMMA 4.3. The solution $\Phi$ whose $\mathrm{p}_{3}$-associate is a spherical harmonic

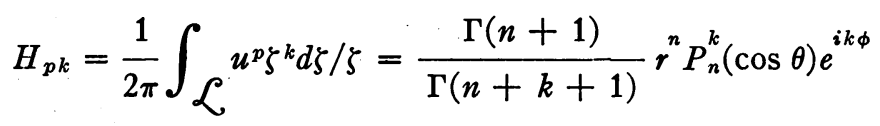

where $r, \theta, \phi$ are polar coordinates, $\mathcal{L}=\mathrm{E}[|\zeta|=1]$, is 


$$
\begin{aligned}
\phi_{p k}(X) & =\mathrm{p}_{3}\left(H_{p k}(X)\right) \\
& =H_{p k}(X)\left[1+\frac{1}{2} \sum_{n=1}^{\infty} \frac{\Gamma(n) \Gamma(p+3 / 2)}{\Gamma(n+p+3 / 2)} B^{(n)}\left(r^{2}\right)\right], \\
r^{2} & =x^{2}+y^{2}+z^{2} .
\end{aligned}
$$

Proof. Relation (4.16) follows by a straightforward computation, if we substitute $H_{p k}(\boldsymbol{X})$ into (4.4) and use the fact that $H_{p k}(\boldsymbol{X})$ is homogeneous.

Every harmonic function in two variables satisfies the Laplace equation in three variables. Thus, considering $h$ and $z^{\prime} h$ where $h=h(Y)$ are rational functions of one complex variable $Y=x^{\prime}+i y^{\prime}$, and $x^{\prime}, y^{\prime}, z^{\prime}$ are new cartesian coordinates obtained by the rotation of the coordinate system, we obtain a class of rational harmonic functions. In considering $\Phi$ with a rational $\mathrm{p}_{3}$ associate, we shall restrict ourselves to the case where the $\mathrm{p}_{3}$-associate is of the form $H(X)=h(Y)$.

Lemma 4.4. The solution $\Phi(X)$ with the $\mathrm{p}_{3}$-associate $(Y-\alpha)^{-1}$ is

$$
\begin{array}{rlr}
\mathrm{p}_{3}\left[(Y-\alpha)^{-1}\right]= & (Y-\alpha)^{-1}+\sum_{n=1}^{\infty} B^{(n)}\left(r^{2}\right) K_{n}(Y), & \\
K_{n}(Y)= & \frac{1}{2}(\alpha / Y)^{1 / 2}\left[\alpha Y^{-1}-1\right]^{n-1} \lg \frac{\alpha^{1 / 2}-Y^{1 / 2}}{\alpha^{1 / 2}+Y^{1 / 2}} & (\text { for } Y \neq 0) \\
& +\sum_{j=0}^{n-1} U_{j} /(1+j) & (\text { for } Y=0) .
\end{array}
$$

Here

$$
U_{j}=S_{j}+\sum_{\lambda=1}^{n-1-\lambda}(-1)^{\lambda}(j / \lambda) C_{j+\lambda-1, \lambda-1}(\alpha / Y) s_{j+\lambda}
$$

and

$$
S_{j}=C_{n-1, j}\left(\alpha Y^{-1}-1\right)^{n-1-j} .
$$

Proof. Substituting $(Y-\alpha)^{-1}$ into (4.12) one obtains (4.17) by a formal computation.

It follows from the representation (4.17) that the function obtained is infinite of the first order along the line $Y=\alpha$, where it has a branch point of infinite order.

In Chapter II we showed that to every R-manifold $R$ given by (2.14) there belongs a family $F$ of algebraic harmonic functions $G(X)=R\left(\zeta^{(\nu)} ; X\right)$ defined on $R$. They are given by (2.13a) and (2.13b). Each of these functions $G \in F$ 
is a rational function of $x, y, z, \zeta^{(v)}$. We proceed now to the study of solutions $\Phi$ which are generated by the above functions $G$.

In order to describe some properties of functions which we obtain in this manner it is useful to consider a new $\mathrm{R}$-manifold which we shall denote by $S$ and which is closely connected with the equation $Q(\zeta ; X)=0$ (see (2.14)). For a fixed value of $\boldsymbol{X}$, the equation

$$
B(\sigma, \zeta ; \boldsymbol{X}) \equiv \zeta^{2 N}+\sum_{\mu=0}^{2 N-1} \frac{A\left(x \sigma^{2}, y \sigma^{2}, z \sigma^{2}\right)}{A_{2 n}\left(x \sigma^{2}, y \sigma^{2}, z \sigma^{2}\right)} \zeta^{\mu}=0
$$

determines a Riemann surface, say $\mathcal{T}(\boldsymbol{X})$, with $2 N$ sheets $\zeta=\zeta^{(\nu)}(\sigma ; X)$, $\nu=1,2, \cdots, 2 N$, which in general has $2 s$ distinct branch points $\sigma^{(\mu)}(X)$, $\mu=1,2, \cdots, 2 s ; 2 s$ being the degree in $\sigma$ of the discriminant $D(\sigma ; X)$ of (4.21). (One obtains $D(\sigma ; \boldsymbol{X})$ by eliminating $\zeta$ from $B(\sigma, \zeta ; \boldsymbol{X})=0$ and $B_{\zeta}(\sigma, \zeta ; X)=0$.) The equation

$$
D(\sigma ; X)=0
$$

defines in the $x, y, z$-space the R-manifold $\mathcal{S}$, mentioned above, which has $2 s$ space sheets, $\sigma^{(\mu)}(X), \mu=1,2, \cdots, s$. We denote by

$$
S^{\prime}=\mathrm{E}\left[D(\sigma ; \boldsymbol{X})=0, D_{\sigma}(\sigma ; \boldsymbol{X})=0\right]
$$

the branchline of $S$.

We wish to indicate some properties of the Riemann surfaces $\mathcal{T}(\boldsymbol{X})$ which we obtain when $X$ ranges over $\mathcal{S}$.

LEMmA 4.5. Let $\mathcal{P}^{\prime}$ be a path on $\mathcal{S}-\mathcal{S}^{\prime}$. The genus $\rho(X)$ of $\mathcal{T}(\boldsymbol{X})$ remains constant when $X$ varies along $\mathcal{P}^{\prime}$.

Proof. Suppose that $\mathcal{L}_{\nu}^{\prime}(\boldsymbol{X}), \mathcal{L}_{\nu}^{\prime \prime}(\boldsymbol{X}), \nu=1,2, \cdots, \rho(\boldsymbol{X})$, form a system of loops and cross sections on $\mathcal{T}(\boldsymbol{X})$ which makes $\mathcal{T}(\boldsymbol{X})$ simply-connected. Since the branchpoints $\sigma^{(v)}(X)$ vary continuously when $X$ varies along $\Phi^{\prime}$ we can deform $\mathcal{L}^{\prime}(\boldsymbol{X})$ and $\mathcal{L}_{\nu}^{\prime \prime}(\boldsymbol{X})$ continuously. Since on the other hand $\rho(X)$ is an integer, it must remain constant, which proves the statement of our lemma.

REMARK 4.2. We note that with every closed oriented curve $N \in S-S^{\prime}$, which cannot be reduced on $\mathcal{S}$ to a point, we can associate the matrix $\mathfrak{p}(\mathcal{N})$ which gives the permutations of the set $\left\{\sigma^{(\nu)}(\boldsymbol{X})\right\}$ while $\boldsymbol{X}$ describes a curve which is homologous to $\mathcal{N}$. These matrices form a group which we shall define as the permutation group of $\mathcal{S}$.

For every $\boldsymbol{X} \in \mathcal{S}-\mathcal{S}^{\prime},\|X\|<\infty$, we form the integrands

$$
H(\sigma, \zeta ; X)_{\alpha}, \quad H^{\prime}(\sigma, \zeta ; X)_{\alpha}, \quad H\left(\sigma, \zeta ; \sigma_{1}, \zeta_{1} ; X\right), \quad \alpha=1,2, \cdots, \rho,
$$

of the first, second, and third kinds, which are defined on $\mathcal{T}(X)$ (see $[15, \mathrm{pp}$. 79 and 73 ]) and the corresponding integrals 


$$
J(\sigma, \zeta ; X)_{\alpha}, \quad J^{\prime}(\sigma, \zeta: X)_{\alpha}, \quad J\left(\sigma, \zeta ; \sigma_{1}, \zeta_{1} ; \sigma_{0}, \zeta_{0} ; X\right) .
$$

(See [15, pp. 373 and 397].)

Let

$$
\omega_{\alpha \beta}(X), \omega_{\alpha \beta}^{\prime}(X) ; \quad \eta_{\alpha \beta}(X), \eta_{\alpha \beta}^{\prime}(X), \quad \alpha=1,2, \cdots, \rho ; \beta=1,2, \cdots, \rho,
$$

denote the periods of the above integrals of the first and second kinds, respectively. (See $[15$, pp. 324].)

Using $\omega_{\alpha \beta}(X)$ and $\omega_{\alpha \beta}^{\prime}(X)$ we form the functions $\theta\left(u_{1}, u_{2}, \cdots, u_{\rho}, X\right.$; $\mu, \mu^{\prime}$ ) (see $[15$, chap. 30]).

Since $\omega_{\alpha \beta}(\boldsymbol{X}), \omega_{\alpha \beta}^{\prime}(\boldsymbol{X})$ depend on $x, y, z$, these functions depend on $\rho+3$ variables, $u_{1}, \cdots, u_{\rho}, x, y, z$. In order to stress the difference from the classiical $\theta$ functions for which the $\omega_{\alpha \beta}, \omega_{\alpha \beta}^{\prime}$ are constants, we shall denote the new functions by $\tilde{\theta}\left(u_{1}, u_{2}, \cdots, u_{\rho} ; X ; \mu, \mu^{\prime}\right)$.

Notation. 4.1. The $\tilde{\theta}\left(u_{1}, \cdots, u_{\rho} ; X ; \mu, \mu^{\prime}\right)$ obtained in the above manner will be defined as $\tilde{\theta}$ functions belonging to the R-manifold $S$. The functions $\tilde{\theta}$ obviously satisfy the relations

$$
\begin{gathered}
\tilde{\theta}\left(u_{1}+2 \omega_{1 \beta}(X), \cdots, u_{\rho}+2 \omega_{\rho \beta}(X) ; X ; \mu, \mu^{\prime}\right) \\
=\exp \left[\mu^{\prime} \beta \pi i\right] \tilde{\theta}\left(u_{1}, \cdots, u_{\rho} ; X ; \mu, \mu^{\prime}\right) \exp \left[\sum_{\alpha} 2 \eta_{\alpha \beta}(X)\left(u_{\alpha}+\omega_{\alpha \beta}(X)\right)\right], \\
(4.27) \quad \tilde{\theta}\left(u_{1}+2 \omega_{1 \beta}(X), \cdots, u_{\rho}+2 \omega_{\rho \beta}^{\prime}(X) ; X ; \mu, \mu^{\prime}\right) \\
=\exp [-\mu \beta \pi i] \tilde{\theta}\left(u_{1}, \cdots, u_{\rho} ; X ; \mu, \mu^{\prime}\right) \\
\cdot \exp \left[\sum_{\alpha} 2 \eta_{\alpha \beta}^{\prime}(X)\left(u+\omega_{\alpha \beta}^{\prime}(X)\right] .\right.
\end{gathered}
$$

See $[15$, p. 576$]$.

The $\tilde{\theta}$ are not single-valued functions of $X$ on $S$. Indeed, suppose that $\mathcal{N}$ is a curve which cannot be reduced to a point, and suppose that when we start from a point $X$, move along $\mathcal{N}$ and return to $X$, the permutation which the crosscuts and loops undergo is

$$
\left(\begin{array}{ccc}
1 & \cdots & \rho \\
\beta_{1} & \cdots & \beta_{\rho}
\end{array}\right) .
$$

The $\omega_{\alpha \beta}(X)$ and $\omega_{\alpha \beta}^{\prime}(X)$ undergo the corresponding permutation and therefore when we describe a circuit along $\mathcal{N}$ we return to a $\tilde{\theta}$ function different from the one from which we started.

Notation. 4.2. Suppose a function $N(X)$ can be represented by using (1) $\tilde{\theta}\left(u_{1}, \cdots, u_{p} ; X ; \mu, \mu^{\prime}\right)$ belonging to the R-manifold $S$; (2) their partial derivatives of the first order with respect to $u_{x}$; (3) transcendental functions $J(\sigma, \zeta ; X)_{\alpha}$ belonging to $\mathcal{S}$, and (4) finitely many algebraic and logarithmic operations. Here, algebraic functions of $x, y, z$ can to be substituted for 
$u_{1}, \cdots, u_{\rho}, \sigma$ and $\zeta$. Such a function will be said to belong to the family $G(\mathcal{S})$.

THEOREM 4.1. Let $F$ be the family of algebraic harmonic functions defined on the R-manifold $R$ given by $A(\zeta ; X)=0$ (see (2.14)). Then the solutions $\Phi=\mathrm{p}_{3}(G)$ of $\mathbf{T}(\Phi)=0$ generated from $G ; G \in F$, can be represented in the form

$$
\Phi(\boldsymbol{X})=G(\boldsymbol{X})+\sum_{n=1}^{\infty} B^{(n)}\left(r^{2}\right) G_{n}(\boldsymbol{X})
$$

where $B^{(n)}\left(r^{2}\right)$ are the coefficients of the operator (and therefore depend only upon $T)$, and $G_{n} \in G(\mathrm{~S})$, S being the R-manifold which we associate with $\mathrm{R}$.

Proof. By (4.1)

$$
G_{n}(X)=\int_{\sigma=0}^{1}\left(1-\sigma^{2}\right)^{n-1} \sigma^{2} G\left(\sigma^{2} x, \sigma^{2} y, \sigma^{2} z\right) d \sigma .
$$

According to (2.13a), (2.13b), (2.14): $G\left(\sigma^{2} X\right)=R(\sigma, \zeta ; X)$ is an algebraic function of $\sigma$ which is defined on the Riemann surface $\mathcal{G}(\boldsymbol{X})$ given by (4.21). Therefore, according to $\left[15\right.$, p. 264] $G\left(\sigma^{2} X\right)$ can be written in the form

$$
\begin{aligned}
G\left(\sigma^{2} \boldsymbol{X}\right) & =\sum_{\nu} c_{\nu}(\boldsymbol{X}) H\left(\sigma, \zeta ; \sigma_{\nu}, \zeta_{\nu} ; \boldsymbol{X}\right) \\
& -\sum_{\alpha=1}^{\rho}\left[g_{\alpha}^{*}(\boldsymbol{X}) H(\sigma, \zeta ; \boldsymbol{X})_{\alpha}+g_{\alpha}(\boldsymbol{X}) H^{\prime}(\sigma, \zeta ; \boldsymbol{X})_{\alpha}\right]+d F(\sigma, \zeta ; \boldsymbol{X}) / d \sigma
\end{aligned}
$$

where $c_{\nu}(\boldsymbol{X}), g_{\alpha}(\boldsymbol{X}), g_{\alpha}^{*}(\boldsymbol{X})$, and $F(\sigma, \zeta ; \boldsymbol{X})$ are functions which are obtained by algebraic operations from coefficients of $G\left(\sigma^{2} X\right)$ and are therefore algebraic functions of $\boldsymbol{X}$. Substituting for $\zeta$ algebraic functions of $\sigma$, and choosing for $\sigma$ suitably chosen fixed values, we obtain the periods (which are functions of $\boldsymbol{X}$ ) of integrals of the first kind.

According to [15, pp. 597 and 598], the integrals of the second and third kind can be expressed in $\tilde{\theta}$ functions, their first partial derivatives, their logarithms, and the periods of the first kind. This completes the proof of Theorem 3.1.

\section{BIBLIOGRAPHY}

1. Stefan Bergman, Zur Theorie der ein- und mehrwertigen harmonischen Funktionen des drei-dimensionalen Raumes, Math. Zeit. vol. 24 (1926) pp. 641-669.

2. - Zur Theorie der algebraischen Potentialfunktionen des drei-dimensionalen Raumes, Math. Ann. vol. 99 (1928) pp. 629-659, and vol. 101 (1929) pp. 534-558.

3. - Zur Theorie der Funktionen, die eine lineare partielle Differentialgleichung befriedigen, Rec. Math. (Mat. Sbornik) N.S. vol. 2 (1937) pp. 1169-1198.

4. - Linear operators in the theory of partial differential equations, Trans. Amer. Math. Soc. vol. 53 (1943) pp. 130-155.

5. - Residue theorems of harmonic functions of three variables, Bull..Amer. Math. Soc. vol. 49 (1943) pp. 163-174.

6. - Solution of linear partial differential equations of the fourth order, Duke Math. J. vol. 11 (1944) pp. 617-649. 
7. - Certain classes of analytic functions of two real variables and their properties, Trans. Amer. Math. Soc. vol. 57 (1945) pp. 299-331.

8. - A class of harmonic functions in three variables and their properties, Trans. Amer. Math. Soc. vol. 59 (1946) pp. 216-247.

9. - Classes of solutions of linear partial differential equations in three variables, Duke Math. J. vol. 13 (1946) pp. 419-458. tion.

10. - On multivalued solutions of differential equations in three variables. In prepara-

11. W. Magnus and F. Oberhettinger, Formeln und Saetze fuer die speziellen Funktionen der mathematischen Physik, Berlin, 1948.

12. R. von Mises, Integral theorems in three-dimensional potential flow, Bull. Amer. Math. Soc. vol. 50 (1944) pp. 599-611.

13. V. Volterra, Sulla integrazione di un sistema di equazioni differenziali a derivate parziali che si presenta nella teoria delle funzione coniugate, Rend. Circ. Mat. Palermo vol. 3 (1889).

14. - Sur une géneralisation de la théorie des fonctions d'une variable complexe, Acta Math. (1889) p. 233.

15. K. Weierstrass, Vorlesungen ueber die Theorie der Abelschen Transcendenten, Gesammelte Werke, Berlin, 1902.

16. H. Weyl, Die Idee der Riemann'schen Flaeche, Leipzig, 1923.

17. M. M. E. Eichler, On the differential equation $u_{x x}+u_{y y}+N(x) u=0$, Trans. Amer. Math. Soc. vol. 65 (1949) pp. 259-278.

18. - Analytic functions in three-dimensional Riemannian spaces, Duke Math. J. vol. 16 (1949) pp. 339-349.

HARVARD UNIVERSITY,

Cambridge, Mass. 